\title{
Photon emission by an ultra-relativistic particle channeling in a periodically bent crystal 由
}

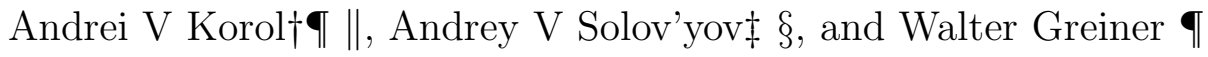 \\ $\dagger$ Department of Physics, St.Petersburg State Maritime Technical University, Leninskii \\ prospect 101, St. Petersburg 198262, Russia \\ $\ddagger$ A.F.Ioffe Physical-Technical Institute of the Academy of Sciences of Russia, \\ Polytechnicheskaya 26, St. Petersburg 194021, Russia \\ ๆInstitut für Theoretische Physik der Johann Wolfgang Goethe-Universität, 60054 \\ Frankfurt am Main, Germany
}

\begin{abstract}
This paper is devoted to a detailed analysis of the new type of the undulator radiation generated by an ultra-relativistic charged particle channeling along a crystal plane, which is periodically bent by a transverse acoustic wave, as well as to the conditions limiting the observation of this phenomenon. This mechanism makes feasible the generation of electromagnetic radiation, both spontaneous and stimulated, emitted in a wide range of the photon energies, from $X$ - up to $\gamma$-rays.
\end{abstract}

PACS numbers: 41.60

\section{Introduction}

This paper is devoted to a detailed analysis of the new type of the undulator radiation (acoustically induced radiation - AIR) generated by an ultra-relativistic charged particle channeling along a crystal plane, which is periodically bent by a transverse acoustic wave (AW), as well as to the conditions limiting the observation of this phenomenon. This mechanism, suggested recently in [1, 2], makes feasible the generation of electromagnetic radiation, both spontaneous and stimulated, emitted in a wide range of the photon energies, from $X$ - up to $\gamma$-rays.

The mechanism of the AIR generation is illustrated in figure 1. Under the action of a transverse acoustic wave propagating along the $z$-direction, which defines the center line of an initially straight channel (not plotted in the figure) the channel becomes

$\ddagger$ published in Int. J. Mod. Phys. E, Vol. 8, No. 1 (February 1999) 49-100

|| E-mail: korol@rpro.ioffe.rssi.ru, korol@th.physik.uni-frankfurt.de

$\S$ E-mail: solovyov@rpro.ioffe.rssi.ru 
periodically bent. Provided certain conditions are fulfilled (see below in this section and in section 3), the beam of positrons, which enters the crystal at a small incident angle with respect to the curved crystallographic plane, will penetrate through the crystal following the bendings of its channel. It results in the transverse oscillations of the beam particles while travelling along the $z$ axis. These oscillations become an effective source of spontaneous radiation of undulator type due to the constructive interference of the photons emitted from similar parts of the trajectory. As we demonstrate below, the number of oscillations can vary in a wide range from a few up to a few thousands per $\mathrm{cm}$ depending on the the beam energy, the AW amplitude, $a$, and wavelength, $\lambda$, the type of the crystal and the crystallographic plane. In addition to the spontaneous photon emission by the undulator, the scheme presented in figure 1 leads to a possibility to generate stimulated emission. This is due to the fact, that photons, emitted at the points of the maximum curvature of the trajectory, travel almost parallel to the beam and, thus, stimulate the photon generation in the vicinity of all successive maxima and minima of the trajectory.

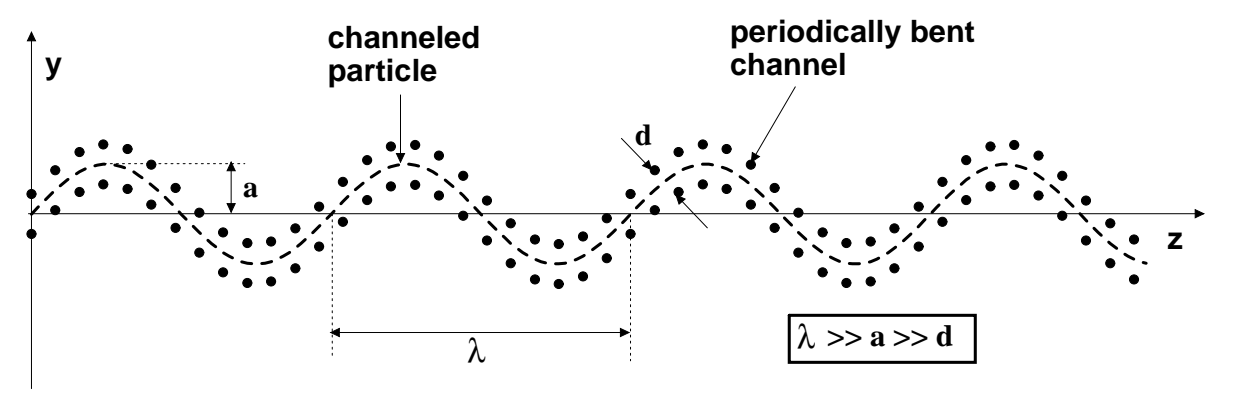

Figure 1. Schematic representation of the initially linear planar channel bent by the transverse acoustic wave. The notations are: $d$ is the channel width, $a, \lambda$ are the AW amplitude and wavelength, respectively.

Some aspects of the electromagnetic radiation by a beam of charged particles channeling in a crystal undergoing the action of an ultrasonic wave have been discussed in the literature [3]-9]. We shall mention the main results obtained in the cited papers and outline the features, both of formal and of principal nature, which distinguish the AIR radiation described above from the effects considered in [3]-[9] at the end of this section.

Prior to that let us briefly discuss the phenomena closely related to the mechanism of generating the acoustically induced radiation. These are the channeling effect, the channeling radiation and the undulator radiation.

The basic effect of the channeling process in a straight crystal is that a charged particle can penetrate an anomalously large distance inside a crystal if travelling nearly parallel to the crystallographic plane or axis and experiencing the collective action of the electric field of the lattice ions. Positively charged particles are steered into the 
interatomic region (the planar channeling) while negatively charged projectiles move in vicinity of the ionic chains (the axial channeling).

Channeling was discovered in the early 1960s by computer simulations of ion motion in crystals [10, 11]. Large penetration lengths were obtained for ions incident along crystallographic directions of low indexes. It is interesting to note that such a guided motion had already been predicted [12] by Stark in 1912 [12]. A comprehensive theoretical study [14, 13] introduced the important continuum approximation for the interaction potentials between energetic charged projectiles and lattice atoms arranged in atomic strings and planes. These concepts were subsequently widely used in the interpretation of channeling experiments (see e.g. the review article [15]). During recent years particular attention has been paid to studying of the channeling phenomenon at high energies of the particles [16, 17, 18]).

The idea of bending the high-energy beams of charged particles, proposed in [19], has become of great current interest [16, 18, [20]- [24], 25] because of its practical application for the manipulation of the beams. Indeed, the high-energy beams can be bent by crystals as efficiently as by means of external electric or magnetic fields. The size of such crystals is typically hundreds times smaller than the size of the equivalent magnet. In recent experiments with $450 \mathrm{GeV}$ protons 23] the efficiency of the particle beam deflection was reported on the level of $60 \%$.

Another possible application of bent crystal was suggested in [26], where the question whether the channeling effect can also be used to focus beams, especially heavy-ion beams, was discussed. To this end a crystal is needed in which the crystal axes are no longer parallel, but are slanted more and more the farther away they are from the axis of the beam. Then the bending angle of the particles far away from the beam axis would be largest and a general focusing effect will result. Such a crystal can in principle be produced by varying the germanium to silicon ratio in a mixed crystal [26].

The criterion for a stable channeling of an ultra-relativistic particle in a bent crystal was formulated in [19] and has clear physical meaning: the maximum centrifugal force due to the channel bending must be less than that of the interplanar field. Provided this condition is fulfilled, the beam of (positively charged) channeling particles at each instant moves inside the channel, especially parallel to the bent crystal midplane as it does in the case of linear channeling.

Two realistic ways of "preparation" of a periodically bent channel may be discussed. It is feasible, by means of mordern technology [25], to grow the crystal with its channels been statically bent according to a particular pattern. The channeling phenomenon has been also considered in the periodic structures such as superlattices [5, 6]. THe possibility [1, 2], [3] - [9], of a dynamically bent crystal, arises if one considers propagation of an acoustic wave along some particular direction in a crystal as shown in figure 1. Under the action of the AW the crystal channels, being linear initially, 
will be periodically bent. In both cases the passage of an ultra-relativistic particle along the bent channel gives rise to the AIR phenomenon due to the curvature of the trajectory, provided that the projectile is trapped into the channel. The latter condition is subject to the general criterion for the channeling process in a bent crystal [19] (see also [3, 25, 27]), and can be fulfilled by a proper choice of projectile energy and maximal curvature of the channel, as described below in the paper.

The advantage of the static channel is that its parameters are fixed and, thus, the projectile moves along the fixed trajectory as well. To calculate the characteristics of the emitted radiation one needs to know only the number of the periods and the local cuvature radius. The disadvantage is that when fixing the number of periods then the parameters of the system can be varied only by changing the energy of the particle. This makes the photon generation less tunable.

The motion of a particle in the field of the channel bent by transverse AW becomes periodic and, therefore, the spectrum of radiation emitted by a projectile acquires essential features of the undulator radiation.

It is natural that the AIR emission is accompanied by the ordinary channeling radiation [28, 29]. This specific type of electromagnetic radiation arises due to the transverse motion of the channeling particle inside the channel under the action of the interplanar field. The phenomena of the channeling radiation of a charged projectile in a linear crystal (see eg. [30, 31, 32]) as well as in a "simple" (i.e. non-periodically) bent channel [27, 33], are known, although in the latter case the theoretical and experimental data are scarce, at least up to now.

We demonstrate in our paper that the AIR and the ordinary channeling radiation can be separated provided the condition $a \gg d$ is fulfilled (see figure 1). Then the frequency of the particle oscillations inside the channel is much higher than the frequency of the transverse oscillations caused by the AW. Therefore, these two motions are well separated and the AIR mechanism can be treated independently from the ordinary channeling radiation. We shall discuss this important issue in more detail in section 3 . Here let us only note that a similar situation occurs in a one-arc bent crystal, where the channeling charged particle generates additional synchrotron type radiation due to the curvature of the channel [27, 33]. This component of the total radiation intensity leads to the undulator effect in the channel periodically bent by AW. We will show that the intensity of AIR can be made larger than that of the channeling radiation.

As was pointed out in [1, 2], the system "ultra-relativistic charged particle + periodically bent crystal channel" represents by itself a new type of undulator, and, consequently, serves as a new source of undulator radiation of high intensity, monochromaticity and with a particular pattern of the angular-frequency distribution. The electromagnetic radiation in this undulator arises mainly due to the bending of the particle trajectory which follows the shape of the channel. The parameters of this 
undulator, as well as the characteristics of the electromagnetic radiation, depend on the type of the crystal and on the crystallographic plane, on the type of projectile and its energy; they also depend on the shape of the bent channel, and, thus, can be varied significantly by varying the enlisted characteristics.

The theory and also the various practical implementations of the undulator radiation, e.g. the radiation emitted by a charge moving in spatially periodic static magnetic fields (a magnetic undulator), in a laser field (a laser-based undulator), etc. have a long history [34, 35] and are well elaborated [30, 31, 36, 37]. It is also known [38] that a positively charged ultra-relativistic particle, undergoing a planar channeling in a linear crystal, radiates electromagnetic waves whose spectral and angular distributions are those of the undulator (a natural undulator) due to the transverse oscillations caused by the action of the repulsive interplanar potential. Also the characteristics of the radiation emitted in a natural undulator are very sensitive to the distribution of the particles in the beam over the transverse energy and the incident angle.

The important feature of the dynamically bent crystal by means of an AW is that it allows to consider an undulator with the parameters $N_{\mathrm{u}}$ and $p$ (here $N_{\mathrm{u}}$ is the number of periods in the undulator and $p=2 \pi \gamma a / \lambda$ is its parameter (see figure 1), and $\gamma$ is the relativistic factor) varying over a wide range, which is determined not only by the projectile's energy but also by the AW frequency and amplitude. The latter two quantities can easily be tuned resulting in the possibility of significantly varying the intensity and shape of the angular distribution of the radiation. It is important to note that the parameters of the acoustically based undulator are inaccessible in the undulators based on the motion of charged particles in periodic magnetic fields and also in the field of laser radiation [30, 36, 39].

In the suggested scheme AIR is generated by the relativistic charged particles, with relativistic factors $\gamma=\varepsilon / m c^{2} \gg 1$ ( $c$ is the velocity of light, $m$ is the mass of the particle and $\varepsilon$ is its energy). The large range of $\gamma$ available in modern colliders for various charged particles, both light and heavy, together with the wide range of frequencies and the amplitudes possible for AW in crystals allow to generate AIR photons with the energies up into the $\mathrm{GeV}$ region [1]].

As demonstrated in [2], the specific pattern of the undulator radiation combined with the AIR mechanism allows to discuss the possibility to create a powerful source of stimulated monochromatic radiation of high-energy photons. We analyse this important possibility in more detail in the present article. Particular attention is paid to the investigation of the influence of various physical processes, such as the photon attenuation and the dechanneling effect of positrons in crystals, both leading to a reduction in the amplification of the stimulated photon emission.

Let us briefly outline here the assumptions, which are adopted in this paper and formulate the main conditions which allow to consider the AW bent crystal as an 
undulator.

Although the general treatment of both the undulator radiation (section 2) and the channeling process in the AW bent crystal (section 3) can be applied to axial and a planar channeling, we consider the latter case because it is known (see e.g. [25]) that for bent crystals negatively charged projectiles are steered along crystallographic axes much less efficiently than positively charged ones along the crystal planes. In fact, experimentally the axial channeling of negative particles has not been observed so far.

Two important conditions which we assume to be fulfilled are the following:

a) The bending of the channel becomes significant if the AW amplitude is noticeably larger than the interplanar spacing, $d$ (see figure 1). Thus, the strong inequality

$$
a \gg d
$$

is implied throughout the paper. Typically, the spacing between the planes, which are characterized by the low values of the Miller indices (such as the (100), (110) and (111) planes), lies within the range $0.6 \ldots 2.5 \AA$ (see e.g. [25] and table 1 in section 5) below. Therefore, (11) is fulfilled for the AW amplitudes $a \geq 10 \AA$.

b) The channeling process in a bent crystal takes place if the maximal centrifugal force in the channel, $m \gamma v^{2} / R_{\min }$ (where $R_{\min }$ is a minimum curvature radius of the bent channel) is less than the maximal force due to the interplanar field [19, 3, 40, 27]:

$$
m \gamma v^{2} / R_{\min }<q U_{\max }^{\prime}
$$

Here $q$ is the charge of the projectile and the quantity $U_{\max }^{\prime}$ stands for the maximum gradient of the interplanar field.

Provided (2) is fulfilled, the projectile, incident at small angle with respect to the mid-plane, will be trapped in the channel (see figure 1). The condition (1) allows to disregard the oscillations of the particle inside the channel due to the interplanar force $q U^{\prime}$. Therefore, the particle trajectory will be primarily harmonic defined by the equation

$$
y(z)=a \sin \left(2 \pi \frac{z}{\lambda}\right), \quad z=[0 \ldots L]
$$

Here $L$ stands for the thickness of the crystal. The minimum curvature radius of this trajectory is equal to $R_{\min }=(\lambda / 2 \pi)^{2} / a$. Thus, the decrease in $R_{\min }$ and, consequently, the increase in the maximum acceleration of the particle in the channel is achieved by decreasing $\lambda$ and increasing $a$. As a result, photon emission due to the projectile's acceleration in the bent channel may be significantly enhanced. This radiation is emitted coherently from similar parts of the trajectory, and may dominate [1] over the channeling radiation. 
An adequate approach to the problem of the radiation emission by an ultrarelativistic particle moving in an external field was developed by Baier and Katkov in the late 1960s 41 and was called by the authors the "operator quasi-classical method". The details of that formalism can be found in [31, 42] and will not be reproduced here.

The advantage of this method is that it allows to use the classical trajectory for the particle in an external field and, simultaneously, it takes into account the effect of the radiative recoil.

The classical description of the particle motion is valid provided the characteristic energy of the projectile in an external field, $\hbar \tilde{\omega}_{o}$, is much less than its total energy, $\varepsilon=m \gamma c^{2}$. The relation $\hbar \tilde{\omega}_{o} / \varepsilon \propto \gamma^{-1} \ll 1$ is fully applicable in the case of an ultrarelativistic projectile. Typical values of $\hbar \omega_{o}$ are in the order of magnitude equal to the continuous potential-well depth, vary from several $\mathrm{eV}$ for the crystals of light materials (e.q. the $\mathrm{LiH}$ crystal [43) up to $10^{2} \mathrm{eV}$ for the crystals of heavy materials (e.g. the $W$ crystal [25).

The role of the radiative recoil, i.e. the change of the projectile energy due to photon emission, is governed by the ratio $\hbar \omega / \varepsilon$. In the limit $\hbar \omega / \varepsilon \ll 1$ a purely classical description [44] of the radiative process can be used. For $\hbar \omega / \varepsilon \leq 1$ the quantum corrections due to the emission of the photon must be taken into account.

The quasi-classical approach neglects the $\hbar \omega_{o} / \varepsilon$ terms, but it explicitly takes into account the quantum corrections due to the radiative recoil in the whole range of the emitted photon energies, except for the extreme high energy tail of the spectrum.

Using this method the spectra of photons and electron-positron pairs in linear crystals were successfully described [31]. It was also applied to the problem of synchrotron-type radiation emitted by an ultra-relativistic projectile channeling in a non-periodically bent crystal [27, 33].

We use this general formalism of Baier and Katkov in our paper to treat the AIR phenomenon.

To conclude the introductory section let us discuss the results of the papers [3][9] where the electromagnetic radiation emitted by a charge passing through a crystal undergoing the action of an ultrasonic wave was considered.

As far as we are aware of, the first study of the influence of an external ultrasonic field on the radiation of channeling particles in a crystal was given in [3]. In this article (see also [4) spontaneous emission by channeling particles in a silicon crystal bent by an acoustic wave of a frequency $\approx 10 \mathrm{MHz}$ was briefly considered. The estimates carried out in [3, 团 were based on the so-called dipole approximation for the undulator radiation (see e.g. [30]). The latter implies that the strong inequality, $p \ll 2 \pi \gamma a / \lambda \ll 1$ is valid, so that all the undulator emission occurs in the fundamental harmonic. Such an assumption leads to a considerable narrowing of the parameters of the AIR radiation and, also, disregards the possibility to generate the emission in higher harmonics. 
In papers [7, 9] the spontaneous channeling radiation in the presence of a lowamplitude ultrasonic wave was considered by means of classical electrodynamics. In this limit the AW amplitude is much less than the interplanar spacing, $a \ll d$, resulting, thus, in low values of the curvatures of the acoustically bent channels. This inequality allowed the authors of [7, 9] (see also [5]) to consider the phenomenon of resonant enhancement of the photon yield due to the coupling of two mechnisms of the photon emission, the channeling radiation [28, 29] and that induced by the AW.

In [6] both the spontaneous undulator emission and that of a free electron laser type was investigated in the case of a relativistic positron beam channeling through a periodically strained lattice. In this paper the main attention was paid to the radiation emitted in an undulator based on a solid state superlattice (see also [5]). By using the formalism of classical electrodynamics the authors of [6] performed a self-consistent treatment involving the wave equation for the electromagnetic radiation field and the kinetic equation for the positron distribution function. The important result, which is the gain coefficient for the forward radiating field was obtained analytically in a closed

form. Numerical estimates of the gain in the case of a resonant coupling of the channeling radiation and that arising from the superlattice based undulator were presented.

In [6] there is also a short comment on the possibility of using an acoustically bent crystall as an undulator. In our opinion, the analogy between a superlattice and an acoustic wave can not be considered as adequate, because the acoustic wave amplitude, $a$, can greatly exceed the interplanar distance $d$, whereas in the superlattice based undulator the inverse condition is assumed, $a \ll d$. This latter case was analyzed in [5, 6, 7, 9].

It is stressed in figure 1 and in (1) that we consider here the opposite case $a \gg d$. In this limit there is no resonant coupling between the channeling and the undulator radiation because the characteristic frequencies of the photons emitted via these mechanisms are totally incomparable (see section 3). As a result it is possible to distinguish two mechanisms of the radiation formation in an acoustically bent crystal and to investigate the properties of the AIR, both spontaneous and stimulated, separately from the channeling radiation.

As we demonstrate below, staying within the regime defined by (1), it is possible, by tuning the AW amplitude or frequency, to vary significantly the intensity of the AIR radiation, to change the patterns of its spectral and angular distributions. These characteristics of the radiation, as it is known from the general theory of undulators (e.g. 31]), are crucially dependent on the magnitude of the undulator parameter $p$. In our paper we provide the analysis of the AIR radiation for both limiting cases, $p^{2} \ll 1$ and $p>1$.

In the case $a \gg d$ it is important to establish the conditions under which the acoustically bent crystal may serve as an undulator for the channeled ultra- 
relativistic particle. A comprehensive analysis of these conditions, and consequently, the establishment of the ranges of all parameters in the problem (these are: the AW amplitude, frequency and velocity, the type of projectile and its energy, the width of the channel and the magnitude of the interplanar field) inside which the AIR process is feasible, are studied in our paper. In contrast such a discussion is fully omitted in the papers cite above.

Another point we wish to emphasis is that the quasi-classical approach used in our paper is more appropriate than the purely classical treatment utilized in the cited papers. In our paper we consider the spectral distribution of the radiation in the whole range of the photon energies: $\hbar \omega=0 \ldots \varepsilon$. It is clear that the classical approach is valid only in the soft-photon limit, i.e. $\hbar \omega \ll \varepsilon$, but is totally inappropriate in the case $\hbar \omega \lesssim \varepsilon$ where the quantum corrections (the radiative recoil) must be taken into account. The emission of high-energy photons becomes important for energies of projectile positrons in the tens $\mathrm{GeV}$ range.

In our paper it is demonstrated, for the first time, that it is feasible to consider, by means of the AIR mechanism, stimulated emission within the $10^{-2} \ldots 10^{0} \mathrm{MeV}$ range. To establish this interval we carried out a realistic analysis of the influence of the dechanneling and the photon attenuation effects on the spectrum of the AIR radiation.

All the features mentioned above distinguish the results presented below from the earlier works [3]-[7], 9].

Finally, let us note that we do not consider the electromagnetic radiation [8] arising during the projectile channeling in an ultrasonically excited crystal due to specific diffraction of the photons by sets of periodically bent planes.

The paper is organized as follows:

In section 2 the general formalism of the quasi-classical description of the undulator radiation is presented. Section 3 is devoted to a detailed analysis of the conditions which allow to consider the acoustically bent crystal as an undulator. Numerical results for the angular and spectral distributions of the undulator radiation as well as the energy loss due to the AIR are presented in section 0 for various crystals and projectile energies. In section 5 the estimates, both analytical and numerical, for the possibility to generate the stimulated emission by means of the acoustically based undulator are presented. In section 6 we outline the relevant problems which, to our mind, deserve to be thoroughly investigated.

\section{Quasi-classical description of the undulator radiation}

Within the framework of the quasi-classical approach the distribution of the energy radiated in given direction by an ultra-relativistic particle (of a spin $s=1 / 2$ ) and summed over the polarizations of the photon and the projectile, is given by the following 
expression

$$
\mathrm{d} E_{\omega}(\mathbf{n})=\left(\frac{q c}{2 \pi}\right)^{2} \mathrm{~d} \mathbf{k} \int \mathrm{d} t_{1} \mathrm{~d} t_{2} \mathrm{e}^{\mathrm{i} \omega^{\prime} \varphi\left(t_{1}, t_{2}\right)} f\left(t_{1}, t_{2}\right)
$$

Here $\mathrm{d} \mathbf{k}=c^{-3} \omega^{2} \mathrm{~d} \omega \mathrm{d} \Omega_{\mathbf{n}}, \mathbf{n}=c \mathbf{k} / \omega$ is the unit vector in the direction of the photon emission, $c$ is the light velocity and $q$ is the projectile charge. The functions $\varphi\left(t_{1}, t_{2}\right)$ and $f\left(t_{1}, t_{2}\right)$ are defined as follows

$$
\begin{aligned}
& \varphi\left(t_{1}, t_{2}\right)=t_{1}-t_{2}-\frac{1}{c} \mathbf{n} \cdot\left(\mathbf{r}_{1}-\mathbf{r}_{2}\right) \\
& f\left(t_{1}, t_{2}\right)=\frac{1}{2}\left\{\left(1+(1+u)^{2}\right)\left(\frac{\mathbf{v}_{1} \cdot \mathbf{v}_{2}}{c^{2}}-1\right)+\frac{u^{2}}{\gamma^{2}}\right\}
\end{aligned}
$$

The notations used are $\mathbf{r}_{1,2}=\mathbf{r}\left(t_{1,2}\right), \mathbf{v}_{1,2}=\mathbf{v}\left(t_{1,2}\right)$, with $\mathbf{r}$ and $\mathbf{v}$ standing for projectile's radius vector and velocity, respectively.

Expression (14) looks almost like the classical formula [30, 31], although with quantum corrections:

$$
\omega \longrightarrow \omega^{\prime}=\frac{\varepsilon}{\varepsilon-\hbar \omega} \omega, \quad u=\frac{\hbar \omega}{\varepsilon-\hbar \omega},
$$

which take into account the radiative recoil.

Let us now obtain a general quasi-classical expression for the radiated energy, $\mathrm{d} E_{\omega}(\mathbf{n})$, in the case of a particle moving in an undulator which contains $N_{\mathrm{u}}$ periods. The radius vector and the velocity of the particle in an undulator satisfy the conditions

$$
\mathbf{r}(t+T)=\mathbf{r}(t)+\mathbf{v}_{0} T, \quad \mathbf{v}(t+T)=\mathbf{v}(t),
$$

where $T$ is the time interval during which a projectile passes one period of the undulator, and the quantity $\mathbf{v}_{0}$

$$
\mathbf{v}_{0}=\frac{1}{T} \int_{0}^{T} \mathbf{v}(t) \mathrm{d} t
$$

is the mean velocity along the undulator axis.

Following [31] and introducing the quantities

$\mathbf{v}_{T}=\int_{0}^{T} \mathrm{~d} t \frac{\mathbf{v}(t)}{c} \exp (\mathrm{i} \Phi(t)), \quad \mathrm{v}_{T}^{0}=\int_{0}^{T} \mathrm{~d} t \exp (\mathrm{i} \Phi(t)), \quad \Phi(t)=\omega^{\prime}\left(t-\frac{\mathbf{n} \cdot \mathbf{r}(t)}{c}\right)$

expression (雨) can be presented in the form:

$$
\frac{\mathrm{d} E_{\omega}(\mathbf{n})}{\mathrm{d} \omega \mathrm{d} \Omega_{\mathbf{n}}}=\frac{q^{2} \omega^{2}}{8 \pi^{2} c} N_{\mathrm{u}}^{2} D(\eta)\left\{\left(1+(1+u)^{2}\right)\left(\left|\mathbf{v}_{T}\right|^{2}-\left|\mathrm{v}_{T}^{0}\right|^{2}\right)+\frac{u^{2}}{\gamma^{2}}\left|\mathrm{v}_{T}^{0}\right|^{2}\right\}
$$

where the function $D(\eta)$ and its argument are

$$
D(\eta)=\left(\frac{\sin N_{\mathrm{u}} \pi \eta}{N_{\mathrm{u}} \sin \pi \eta}\right)^{2}, \quad \eta=\frac{\omega^{\prime}}{\omega_{0}}\left(1-\frac{\mathbf{n} \cdot \mathbf{v}_{0}}{c}\right),
$$


respectively. The parameter $\omega_{0}=2 \pi / T$ is for the undulator frequency.

Expression (10) together with the definitons (11) clearly exhibit the features typical for the undulator radiation [36]. The radiation intensity is proportional to the square of the total number of the undulator periods, $N_{\mathrm{u}}^{2}$, reflecting the coherence effect of radiation.

The spectral and angular dependence of the radiation are determined mainly by the function $D(\eta)$, which is well-known in a classical theory of diffraction 442. This function has sharp maxima at the points $\eta=K=1,2,3 \ldots$ It is most clearly seen in the case of infinite $N_{\mathrm{u}}$ :

$$
\lim _{N_{\mathrm{u}} \rightarrow \infty} N_{\mathrm{u}} D(\eta)=\sum_{K} \delta(\eta-K)
$$

For $N_{\mathrm{u}}<\infty$ the function $D(\eta)$ has main maxima in the points $\eta=K=1,2 \ldots$ where its magnitude $D(k)=1$. In the interval $\eta=[K, K+1] D(\eta)$ has minima in the points $\eta_{m}^{\min }=K+m / N_{\mathrm{u}}, m=1, \ldots, N_{\mathrm{u}}-1$ where $D\left(\eta_{m}^{\min }\right)=0$. The local maxima are located at $\eta_{m}^{\max }\left(m=1, \ldots, N_{\mathrm{u}}-1\right)$ which can be found from the equation $N_{\mathrm{u}} \operatorname{tg} \pi \eta N_{\mathrm{u}}=\operatorname{ctg} \pi \eta$. The values $D\left(\eta_{m}^{\max }\right)$ rapidly decrease with $m$ and are much smaller than 1 . For $m=1,2$ the corresponding values are $D\left(\eta_{1}^{\max }\right) \approx 1 / 22, D\left(\eta_{2}^{\max }\right) \approx 1 / 62$. The width $\Delta \eta$ of the main maxima of $D(\eta)$, estimated as $\Delta K=2\left(\eta_{1}^{\min }-K\right)$, is equal to $\Delta K=2 / N_{\mathrm{u}}$ and does not depend on $K$.

Since $\mathrm{d} E_{\omega}(\mathbf{n}) \propto D(\eta)$ the frequency-angular distribution of the radiation is represented by the sets of harmonics $\omega_{K}^{\prime}$ (each of the width $\Delta \omega_{K}^{\prime}$ independent on $K$ ) which are effectively emitted at the angle $\theta$ (measured with respect to the undulator axis)

$$
\omega_{K}^{\prime}=\frac{\omega_{0} K}{\left(1-\frac{\mathbf{n} \cdot \mathbf{v}_{0}}{c}\right)}, \quad \Delta \omega^{\prime}=\frac{2}{N_{\mathrm{u}}} \frac{\omega_{K}^{\prime}}{K} \quad K=1,2,3 \ldots
$$

Let indices $\|$ and $\perp$ indicate, respectively, the parallel and the perpendicular (with respect to the undulator axis) components of the projectile radius-vector and velocity:

$$
\mathbf{r}(\tau)=\mathbf{r}_{\|}(\tau)+\mathbf{r}_{\perp}(\tau), \quad \mathbf{v}(\tau)=\mathbf{v}_{\|}(\tau)+\mathbf{v}_{\perp}(\tau), \quad \tau=[0, T]
$$

For $\gamma \gg 1$ the following relations are valid with the accuracy up to the $\gamma^{-2}$ terms:

$$
\frac{\mathrm{v}_{\|}}{c} \approx 1-\frac{1}{2 \gamma^{2}}\left(1+\frac{\mathrm{v}_{\perp}^{2}}{c^{2}} \gamma^{2}\right), \quad \frac{\overline{\mathrm{v}_{\|}}}{c} \equiv \frac{\mathrm{v}_{0}}{c} \approx 1-\frac{1}{2 \gamma^{2}}\left(1+\frac{\overline{\mathrm{v}_{\perp}^{2}}}{c^{2}} \gamma^{2}\right)
$$

where $\overline{\mathrm{v}_{\perp}^{2}}=T^{-1} \int_{0}^{T} \mathrm{v}_{\perp}^{2}(t) \mathrm{d} t$.

By inserting (15) into (9) and introducing the dimensionless variable $\psi=\omega_{0} \tau$, one obtains

$$
\Phi(\tau) \equiv f(\psi)=\eta \psi+\frac{\omega^{\prime}}{\omega_{0}} \cos \theta \frac{\Delta(\psi)}{2}-\frac{\omega^{\prime}}{c} \mathbf{n} \cdot \mathbf{r}_{\perp}(\tau)
$$




$$
\Delta(\psi)=\int_{0}^{\psi}\left[\frac{\mathrm{v}_{\perp}^{2}}{c^{2}}-\frac{\overline{\mathrm{v}_{\perp}^{2}}}{c^{2}}\right] \mathrm{d} \psi
$$

and in the following expression for $\mathrm{d} E_{\omega}(\mathbf{n})$ :

$\frac{\mathrm{d} E_{\omega}(\mathbf{n})}{\mathrm{d} \omega \mathrm{d} \Omega_{\mathbf{n}}}=\frac{q^{2} N_{\mathrm{u}}^{2}}{4 \pi^{2} c} D(\eta) \frac{\omega^{2}(1+u)}{\omega_{0}^{2} \gamma^{2}}\left\{\gamma^{2}\left(1+\frac{u^{2}}{2(1+u)}\right)\left[\left|\mathbf{I}_{\perp}\right|^{2}-\operatorname{ReI}_{0} \mathrm{I}_{\|}\right]-\left|\mathrm{I}_{0}\right|^{2}\right\}$,

where the quantities $\mathrm{I}_{\perp}, \mathrm{I}_{0}$ and $\mathbf{I}_{\|}$stand for the integrals

$$
\mathrm{I}_{0}=\int_{0}^{2 \pi} \mathrm{e}^{\mathrm{i} f(\psi)} \mathrm{d} \psi, \quad \mathrm{I}_{\|}=\int_{0}^{2 \pi} \frac{\mathrm{v}_{\perp}^{2}}{c^{2}} \mathrm{e}^{\mathrm{i} f(\psi)} \mathrm{d} \psi, \quad \mathbf{I}_{\perp}=\int_{0}^{2 \pi} \frac{\mathbf{v}_{\perp}}{c} \mathrm{e}^{\mathrm{i} f(\psi)} \mathrm{d} \psi
$$

Within the same level of accuracy as in (15) the parameter $\eta$ from (11) reads

$$
\eta=\frac{\omega^{\prime}}{\omega_{0}}\left(\frac{1}{2 \gamma^{2}}+\frac{p^{2}}{4 \gamma^{2}}+\frac{\theta^{2}}{2}\right)=\frac{p^{2}}{4 \gamma^{2}} \frac{\omega^{\prime}}{\omega_{0}}\left(\frac{2}{p^{2}}+1+\frac{\theta^{2}}{\theta_{0}^{2}}\right)
$$

The quantity $p$, introduced here, is called the undulator parameter and is defined by the following relation

$$
p^{2}=2 \gamma^{2} \frac{\overline{\mathrm{v}_{\perp}^{2}}}{c^{2}}
$$

The parameter $\theta_{0}$ is related to $p$ and $\gamma$ through

$$
\theta_{0}^{2}=\frac{p^{2}}{2 \gamma^{2}}=\frac{\overline{\mathrm{v}_{\perp}^{2}}}{c^{2}}
$$

and has the following physical meaning: in the case $p^{2} \gg 1$ (so-called, non-dipole limit), $\theta_{0}$ defines the cone, along the undulator axis, $0 \leq \theta \leq \theta_{0}$, into which the radiation is emitted. For $p^{2}<1$ (the dipole case) the emission effectively occurs into the cone $0 \leq \theta \leq \gamma^{-1}$.

The formalism sketched above will be now applied to a planar harmonic undulator, in which the quasi-periodic trajectory (7) lies in a plane. Let the undulator axis coincide with the $z$-direction and let its plane be the $(y z)$-plane. In this type of an undulator the particle trajectory is purely harmonic and is defined as

$$
y(z)=a \sin k z
$$

We assume that the parameters $a$ and $k$ satisfy the condition $\xi \equiv k a \ll 1$. In the next section, where the undulator based on the acoustic wave transmission in a crystal is described in detail, it is demonstrated that this condition is fulfilled.

For an ultra-relativistic projectile, moving along the trajectory (22) the following formulas are valid for the quantities introduced above (the expressions below are written 
in the lowest orders in $\xi$ and $\gamma^{-1}$ ):

$$
\begin{aligned}
& \mathrm{v}_{\|} \simeq \mathrm{v}_{0} \simeq c, \quad \mathrm{v}_{\perp} \simeq c \xi \cos (k z) \ll \mathrm{v}_{\|}, \quad \overline{\mathrm{v}_{\perp}^{2}} \simeq \xi^{2} \frac{c^{2}}{2}, \\
& \Delta(\psi) \simeq \frac{\xi^{2}}{4} \sin 2 \psi, \quad p^{2}=\gamma^{2} \xi^{2}, \quad \omega_{0}=c k, \\
& f(\psi)=\eta \psi+\frac{\omega^{\prime}}{\omega_{0}} \frac{\theta_{0}^{2}}{4} \sin 2 \psi-\frac{\omega^{\prime}}{\omega_{0}} \sqrt{2} \theta_{0} \theta \cos \varphi \sin \psi .
\end{aligned}
$$

Here $\theta$ and $\varphi$ are the polar and the azimuthal angles of the photon emission, respectively.

By substituting (23a-23d) into (17) and (18) one obtains general quasi-classical expressions for the spectral-angular distribution of the radiation in a planar undulator [31]. These expressions can be simplified in two limiting cases: $p^{2} \ll 1$ and $p^{2} \gg 1$. For the sake of further reference below the corresponding formulas are exhibited.

\subsection{Non-dipole case: $p^{2} \gg 1$}

In the limit $N_{\mathrm{u}}, p^{2} \gg 1$ the spectral-angular distribution of the undulator radiation is given by the following asymptotic expression valid for $\theta<\theta_{0}$ :

$$
\begin{aligned}
\frac{\mathrm{d} E(\mathbf{n})}{\mathrm{d} \omega \mathrm{d} \Omega_{\mathbf{n}}}= & \frac{16 q^{2}}{c} \frac{N_{\mathrm{u}}^{2} \gamma^{2}}{p^{2}} \frac{D(\eta)}{1+u}\left(\frac{4 x^{2}}{\mu}\right)^{2 / 3}\left\{\left[\frac{\theta^{2} \sin ^{2} \varphi}{2 \theta_{0}^{2}}+\Delta \cdot \zeta\left(\frac{\mu}{4 x^{2}}\right)^{1 / 3}\right] \cos ^{2} \beta \operatorname{Ai}^{2}(\zeta)\right. \\
& \left.+(1+\Delta)\left(\frac{\mu}{4 x^{2}}\right)^{1 / 3} \sin ^{2} \beta \operatorname{Ai}^{\prime 2}(\zeta)\right\}
\end{aligned}
$$

where $\operatorname{Ai}(\zeta), \operatorname{Ai}^{\prime}(\zeta)$ are the Airy function and its derivative, respectively, and the abbreviations were introduced

$$
\begin{aligned}
& x=\frac{\omega^{\prime}}{\omega_{0}} \frac{p^{2}}{4 \gamma^{2}}, \quad \zeta=\left(\frac{4 x^{2}}{\mu}\right)^{1 / 3}\left[\frac{1}{p^{2}}+\frac{\theta^{2} \sin ^{2} \varphi}{2 \theta_{0}^{2}}\right], \quad \mu=1-\delta^{2} \\
& \delta=\frac{\theta \cos \varphi}{\sqrt{2} \theta_{0}}, \quad \beta=\eta\left(\frac{\pi}{2}-\arcsin \delta\right)-3 x \delta \mu^{1 / 2}, \quad \Delta=\frac{u^{2}}{2(1+u)}
\end{aligned}
$$

Other notations used in (24) have been defined earlier.

The Airy function and its derivative, both satisfying the conditions $\operatorname{Ai}(\zeta),\left|\operatorname{Ai}^{\prime}(\zeta)\right| \sim$ $1, \quad \zeta \leq 1$ and $\operatorname{Ai}(\zeta),\left|\operatorname{Ai}^{\prime}(\zeta)\right| \longrightarrow 0, \zeta \gg 1$, define the frequency of the radiated intensity maximum, $\omega_{\max }^{\prime}$. For $\omega^{\prime} \gg \omega_{\max }^{\prime}$ the intensity of radiation exponentially decreases. It can be shown that $\omega_{\max }^{\prime} \sim p \gamma^{2} \omega_{0}$. It corresponds to $K_{\max } \sim p^{3}$ which is the largest number of the radiated harmonics.

From (24)-(25) follows (in accordance with general theory of a planar undulator [30, 31, 36]) that only odd harmonics $(K=1,3, \ldots)$ are radiated along the undulator axis in the soft-photon (classical) limit, $\hbar \omega / \varepsilon=0$. Indeed, by putting $\theta=0$ and $u=0$ in (24)-(25) one gets $\left[\mathrm{d} E(\mathbf{n}) / \mathrm{d} \omega \mathrm{d} \Omega_{\mathbf{n}}\right]_{\theta=0} \propto D(\eta) \sin ^{2}(\pi \eta / 2)$. Keeping in mind that $\eta=K \pm 1 / N_{\mathrm{u}}$, one gets $K=1,3, \ldots$. 
Equations (24) and (25) are simplified considerably if $\hbar \omega / \varepsilon=0$. It can be demonstrated that in this case (24) represents by itself the classical result for $\mathrm{d} E(\mathbf{n}) / \mathrm{d} \omega \mathrm{d} \Omega_{\mathbf{n}}$ (see e.g. [30]) written in the limit $p^{2} \gg 1$. It is worth noting that for $\hbar \omega / \varepsilon=0$ the asymptotic expression (24) and the exact classical formula written in terms of Bessel functions produce close results starting with the value $p^{2}=2$.

The spectral intensity of the radiation emitted by a projectile moving along the path (22) in an undulator of the total length $L$ can be obtain by integrating (24) over $\varphi=[0,2 \pi]$ and $\theta=\left[0, \theta_{0}\right]$, or by deducing it from the general expression given in 31 . The result is [1]:

$$
\frac{1}{L} \frac{\mathrm{d} E}{\mathrm{~d} \omega}=\frac{q^{2}}{c} N_{\mathrm{u}} p \frac{\omega^{\prime}}{\omega}\left(G_{1}(s)+\left[1+\frac{u^{2}}{2(1+u)}\right] G_{2}(s)\right)
$$

where the parameter $s$ is defined as $s=\left(\omega^{\prime} / \omega_{0} \gamma^{2} p\right)^{2 / 3}$. The functions $G_{1,2}(s)$ are equal to

$$
\begin{aligned}
& G_{1}(s)=-2 s^{5 / 2} \int_{1}^{\infty} \mathrm{d} x\left[\pi-\arccos \left(1-\frac{2}{x^{3}}\right)\right] \operatorname{Ai}(s x) \\
& G_{2}(s)=-8 s^{1 / 2} \int_{0}^{\infty} \frac{\mathrm{d} x}{(\operatorname{ch} x)^{5 / 3}} \operatorname{Ai}^{\prime}\left(s(\operatorname{ch} x)^{2 / 3}\right)
\end{aligned}
$$

In the extreme limit $p^{2} \longrightarrow \infty$ expressions (24) and (27) reduce to the analogue ones for synchrotron radiation [31.

\subsection{Dipole case: $p^{2} \ll 1$}

The general quasi-classical expression one finds in 31. We will need here its classical limit $\hbar \omega / \varepsilon=0$. The angular distribution of the radiation is given by the following expression [30]

$$
\frac{\mathrm{d} E(\mathbf{n})}{\mathrm{d} \omega \mathrm{d} \Omega_{\mathbf{n}}}=\frac{q^{2}}{c} \frac{N_{\mathrm{u}}^{2} \gamma^{2} p^{2}}{\left(1+(\gamma \theta)^{2}\right)^{2}}\left\{\sin ^{2} \varphi+\cos ^{2} \varphi\left(\frac{1-(\gamma \theta)^{2}}{1+(\gamma \theta)^{2}}\right)^{2}\right\} D(\eta)
$$

Note, that in the limit $p^{2} \ll 1$ the parameter $\eta$ reduces to (see (19)):

$$
\eta \simeq \frac{\omega}{\omega_{0}}\left(\frac{1}{2 \gamma^{2}}+\frac{\theta^{2}}{2}\right), \quad \theta=\left[0, \gamma^{-1}\right]
$$

Expression (28) is written in the lowest order in $p^{2}$ and describes the angular distribution of the first harmonic, $K=1$. Thus $\eta=1 \pm 1 / N_{\mathrm{u}}$ is implied in (28). The higher-harmonics emission is strongly supressed in the limit $p^{2} \ll 1$ [30, 31]. The spectral distribution of the radiation in a dipole case can be obtained by a direct integration of (28) over $\varphi=[0,2 \pi]$ and $\theta=\left[0, \gamma^{-1}\right]$. 


\section{Acoustically bent channel as an undulator}

In this section we formulate the conditions which, in addition to (1) and (2), must be fulfilled to consider the system "AW + channeled particle" as an undulator.

Let us consider the case of planar channeling of an ultra-relativistic positively charged particle in a channel bent by a monochromatic transverse acoustic wave (AW) which is transmitted in the $z$-direction (see figure 1). The shape of the channel centerline is described by the dependence (3).

Another condition which we impose on the AW is

$$
\xi \equiv k a=2 \pi \frac{a}{\lambda} \ll 1
$$

where the quantity $k$ denotes the AW wave number, $k=2 \pi / \lambda$. It will be demonstrated below that the condition (30) holds in all cases which are of interest.

\subsection{Motion of the particle in a channel bent by the $A W$}

Let a particle enter the crystal at the point $z=0$ having a small incident angle with respect to the (curved) crystallographic plane. The corresponding quantitative criterion will be given below.

In the case of small-angle injection one may use the continuous potential approximation to describe the interaction of the particle with the crystallographic plane [15, 25]. This potential, $U(\rho)$ depends only on the transverse displacement, $\rho$, of the particle from the mid-plane of the channel $(|\rho| \leq d / 2)$.

In the case of an ultra-relativistic projectile the classical approach adequately describes the projectile motion, so that one may totally disregard the quantum corrections to the transverse oscillations of the projectile in the channel due to the action of the interplanar potential $U$. The following estimate proves this statement. Let $U_{o}$ denote the depth of the interplanar potential well. The separation of the neibouring energy levels, $\hbar \tilde{\omega}_{o}$, of the transverse oscillations is equal, by the order of magnitude, to $\hbar \tilde{\omega}_{o} \approx \hbar \sqrt{q U^{\prime \prime} / m \gamma} \sim \hbar \sqrt{q U_{o} / d^{2} m \gamma}$. Hence, the number of these levels, estimated as

$$
\frac{U_{o}}{\hbar \omega_{o}} \sim \frac{d}{\lambda_{c}} \sqrt{\frac{q U_{o} \gamma}{m c^{2}}} \propto \sqrt{\gamma}, \quad \lambda_{c}=\frac{\hbar}{m c}
$$

is large when $\gamma \gg 1$, and trajectories can be applied to describe the projectile dynamics.

The particle's trajectory lies in the (yz)-plane and is governed by the equation of motion

$$
\frac{\mathrm{d} \mathbf{p}}{\mathrm{d} t}=-q \operatorname{grad} U
$$

where $\mathbf{p}=m \mathbf{v} \gamma$ is the kinematic momentum, $\gamma^{-2}=\left(1-v_{z}^{2} / c^{2}-v_{y}^{2} / c^{2}\right)$. 
We assume that the dependence $U(\rho)$ on $\rho$ in the case of a bent channel is the same as for a linear one. Below we demonstrate that this assumption is adequate.

The Hamiltonian of the particle, $H=\sqrt{p^{2} c^{2}+m^{2} c^{4}}+q U(\rho)$ does not explicitly depend on $t$, hence, the relation $\gamma=H / m c^{2}=\gamma_{0}\left(1+q U / m c^{2} \gamma_{0}\right)$ is valid, with $\gamma_{0}=\left(1-v^{2} / c^{2}\right)^{-1 / 2}$ and $v$ standing for the initial particle velocity. The condition $q U \ll m c^{2} \gamma_{0}$ allows to consider $\gamma$ as an integral of motion.

To proceed further and to obtain all the relations analytically we consider the harmonic approximation for the interplanar potential, $U(\rho)=\kappa \rho^{2} / 2$.

Taking into account (30) the transverse coordinate $\rho$ satisfies the equality $\rho=$ $(y-a \sin k z)$ which is valid up to terms $\sim \xi$. With the same accuracy the equation of motion (32) reads

$$
\frac{\mathrm{d}}{\mathrm{d} t}(\dot{\rho}+\dot{z} \xi \cos k z)=-\frac{q \kappa}{m \gamma} \rho, \quad \frac{\mathrm{d}^{2} z}{\mathrm{~d} t^{2}}=-\frac{q \kappa}{m \gamma} \xi \cos k z
$$

In lowest order in $\xi$ the system (33) yields $z=v t$ (with $v \approx c$ ) and (3) for the particle trajectory. Within the linear approximation in $\xi$ the second equation (33) defines the deviation from this trajectory through 40]

$$
m \gamma \frac{\mathrm{d}^{2} \rho}{\mathrm{d}^{2} t}=-\frac{p v}{R(z)}-q \kappa \rho
$$

The term $-p v / R(z)$ represents the centrifugal force due to the curvature of the bent channel. $R(z)$ is the curvature radius of the curve (3) and is equal to

$$
R(z)=-\frac{1}{k^{2} a \sin k z}
$$

When writing (35) we neglected the terms $\sim \xi^{2}$ and higher.

Equation (35) combined with the inequalities (10) and (30) justifies the approximation made above (see the paragraph after figure 32) concerning the equivalence of the interplanar potentials in the bent channel and in the linear one. Indeed, the deviation of the function $U(\rho)$ due to the channel curvature is proportional to the ratio $d / R$, which can be neglected: $d / R \propto(d / \lambda)(a / \lambda) \ll \xi^{2} \ll 1$.

The general solution of (34) reads

$$
\rho(z)=\rho_{0} \sin (\chi z+\phi)+\frac{a k^{2}}{\chi^{2}-k^{2}} \sin k z, \quad z=v t
$$

where we abbreviated $\chi^{2}=q \kappa /\left(m \gamma v^{2}\right)$. The first term on the right-hand side of (36) describes the oscillations due to the action of the interplanar force $-q U^{\prime}$ and is responsible for the traditional channeling radiation [28]. The constants $\rho_{0}$ and $\phi$ are subject to the initial conditions of the projectile injection into the channel. In the case when the vector of the initial velocity of the particle is aligned with the tangent of the channel centerline one may put $\rho_{0}=0$, and, consequently, $\rho_{0} \sin (\chi z+\phi) \equiv 0$. 
The second term in (36) corresponds to the oscillations of the projectile in the vicinity of the mid-plane due to the action of the (driving) centrifugal force. The channeling in a bent channel is stable against the driving oscillations provided their amplitude, $a k^{2} /\left(\chi^{2}-k^{2}\right)$, is less than half the width of the channel:

$$
\frac{a k^{2}}{\chi^{2}-k^{2}} \ll \frac{d}{2}
$$

Let us introduce a minimum curvature radius of the channel bent by the transverse AW

$$
R_{\min }=\frac{1}{a k^{2}}=\left(\frac{\lambda}{2 \pi}\right)^{2} \frac{1}{a}=\left(\frac{V}{2 \pi}\right)^{2} \frac{1}{\nu^{2} a}
$$

Here $V$ and $\nu$ are the velocity and the frequency of the AW, respectively.

Recalling (1), the condition (37) is presented in the form equivalent to (2):

$$
\frac{\varepsilon}{R_{\min } q U_{\max }^{\prime}}<1
$$

Here the notation $U_{\max }^{\prime}$ is used to substitute $2 \kappa(d / 2)$, and we have put $p v \approx p c=\varepsilon$.

The relation (39) is written in the form which can be used for an arbitrary potential $U(\rho)$. It establishes the condition for a channeling process in a bent channel [19]: the maximal centrifugal force, $\varepsilon / R_{\min }$, must be less than the maximal interplanar force $q U_{\max }^{\prime}$.

The parameter on the left-hand side in (39) defines also a critical incident angle $\Theta_{c}$ 25] of the particle injection into acoustically bent crystal: $\Theta_{\mathrm{c}}=\Theta_{\mathrm{L}}\left(1-\varepsilon / R_{\min } q U_{\max }^{\prime}\right)$, where $\Theta_{\mathrm{L}}=\sqrt{2 q U_{\max } / m \gamma c^{2}}$ is the critical angle for the linear channel [14].

Provided (11) and (39) are fulfilled, the projectile, entering the crystal under an angle $\Theta<\Theta_{c}$ (with respect to the centerline) will be trapped in the channel and move along the trajectory defined by (3). The deviation, (36), of the real trajectory from (3) can be neglected.

By taking into account the last equality from (38) the condition (39) may also be denoted as

$$
\nu^{2} a<C \equiv \gamma^{-1} \cdot\left(\frac{V}{2 \pi}\right)^{2} \cdot\left(\frac{q U_{\max }^{\prime}}{m c^{2}}\right)
$$

and determines the ranges of $\nu=V / \lambda$ ( $\nu$ is the AW frequency), $a$ and $\gamma$ for which the channeling process as well as the undulator radiation, can occur for a given crystal and crystallographic plane (the parameters $U_{\max }^{\prime}$ and $V$ depend on the choice of the particular crystal and the crystallographic plane) and for given projectile type, characterized by its rest mass $m$ and charge $q$.

Figures 2 illustrate the ranges of $\nu$ and $a$ in which the channeling process is possible for a positron and a proton both of the same energy (as indicated) in $C$ (diamond), Si, Ge and $W$ crystals near the (110) crystallographic plane. The solid 
thick lines represent the boundary $\nu^{2} a=C$, so that the range of validity of (40) lies below these lines. To obtain $V$ we used its relation [46] to the crystal elastic constants $c_{11}$ and $c_{12}$ and the density $\rho: V=\sqrt{\left(c_{11}-c_{12}\right) / 2 \rho}$. Using the data from [46] ones gets the following values of $V$ for $C, S i, G e$ and $W$ crystals respectively: $V=11.6 \cdot 10^{5}, 4.67 \cdot 10^{5}, 2.76 \cdot 10^{5}, 2.81 \cdot 10^{5}, \mathrm{~cm} / \mathrm{s}$.

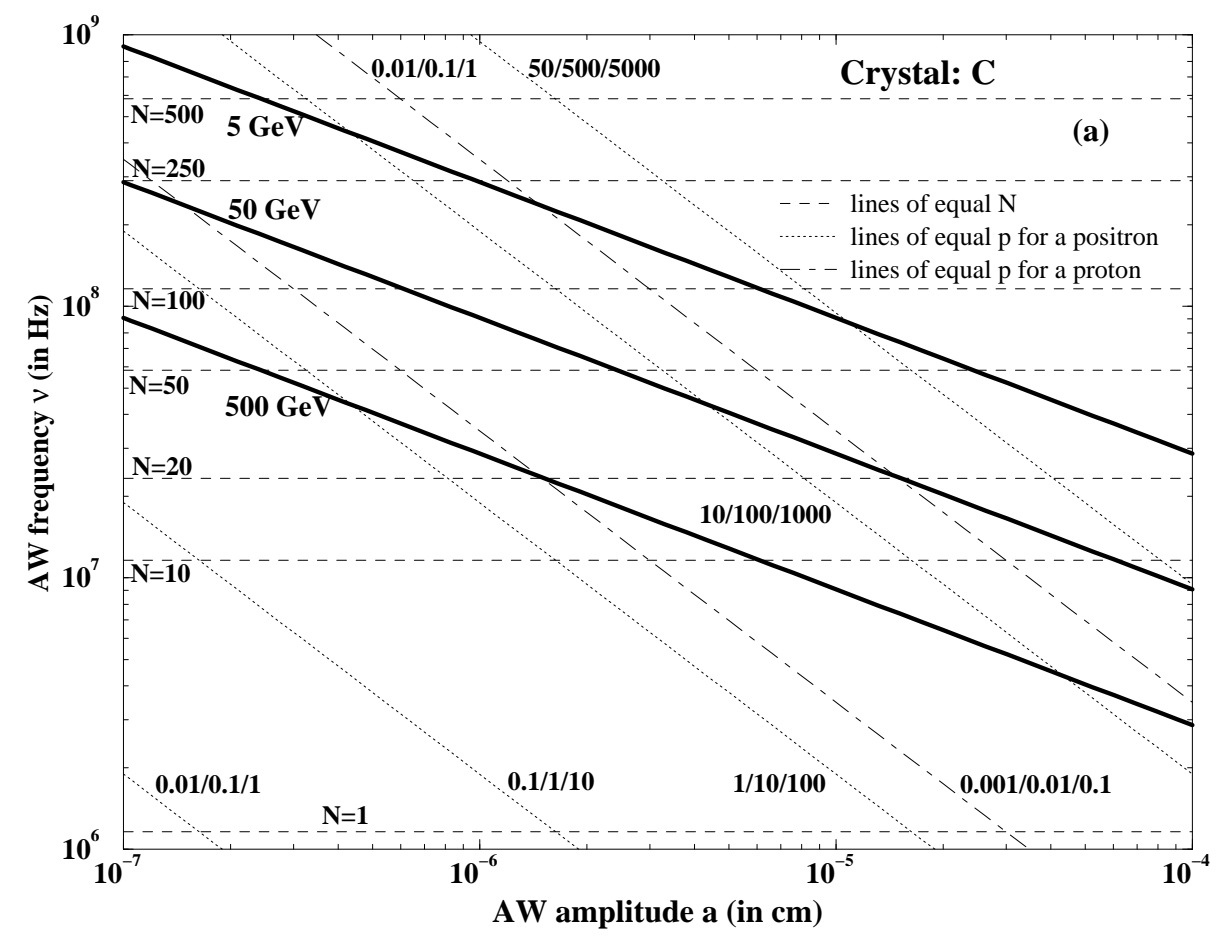

Figure 2. The ranges of $\nu$ (in $\mathrm{Hz}$ ) and $a$ (in $\mathrm{cm}$ ) in which the channelling process is possible for a positron and proton along the (110) plane in (a) a diamond, (b) a silicon crystal, (c) a germanium crystal, (d) a tungsten crystal. The heavy full lines represent the boundaries, $\nu^{2} a=C$ (see (40)), for a $5 / 50 / 500 \mathrm{GeV}$ projectile as indicated. The dashed lines correspond to the constant values of the parameter $N\left(\mathrm{~cm}^{-1}\right)=\lambda^{-1}$ (see also the explanations in the text). The dotted and the chain lines indicate the constant values of the undulator parameter $p=2 \pi \gamma a \nu / V$ for a positron and a proton, respectively. The values of $p, p_{1}, p_{2}$ and $p_{3}$ correspond to the projectile energies 5,50 and $500 \mathrm{GeV}$, respectively.

The data for $q U_{\max }^{\prime}$ (for a positron) are $q U_{\max }^{\prime}=12.0,5.7,10.4,43.0 \mathrm{Gev} / \mathrm{cm}$, and for the interplanar distance $d=1.26,1.92,2.00,2.2410^{-8} \mathrm{~cm}$ for $C$, Si, Ge and $W$ crystals, respectively. They were taken from 25, 31.

Taking into account the double-log scale in these figures it may be concluded, that the channeling condition (40) is fulfilled within wide ranges of the AW amplitudes and frequencies, for different types of projectiles and wide ranges of their energies, and for a variety of crystals. One can also deduce from figures 2 that within the range of validity of (40) the condition (30) is well fulfilled. 


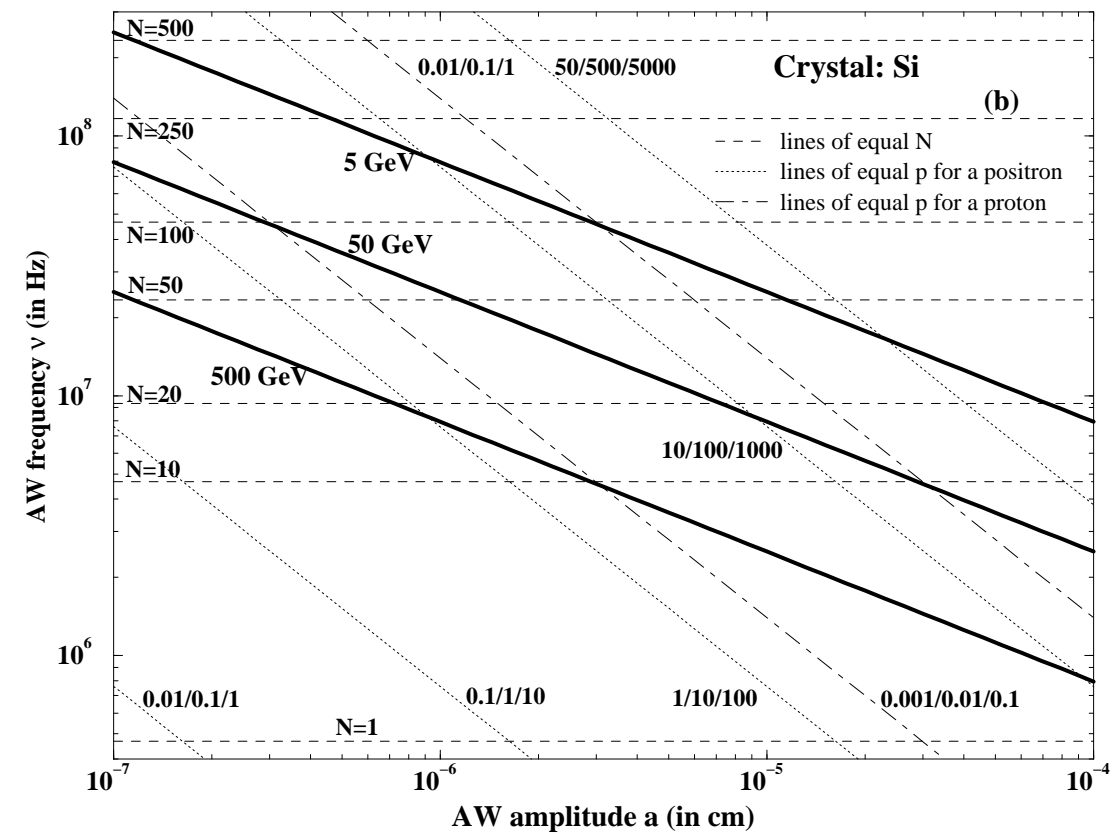

Figure 2. (continued)

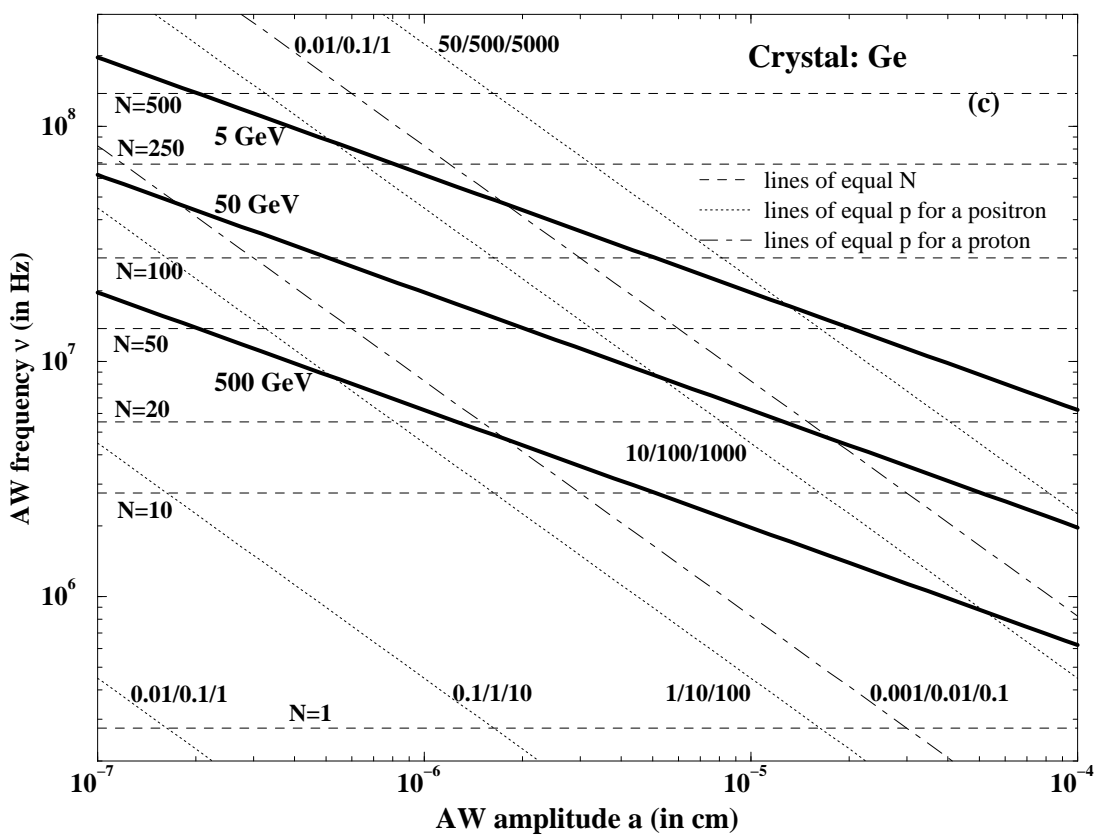

Figure 2. (continued) 


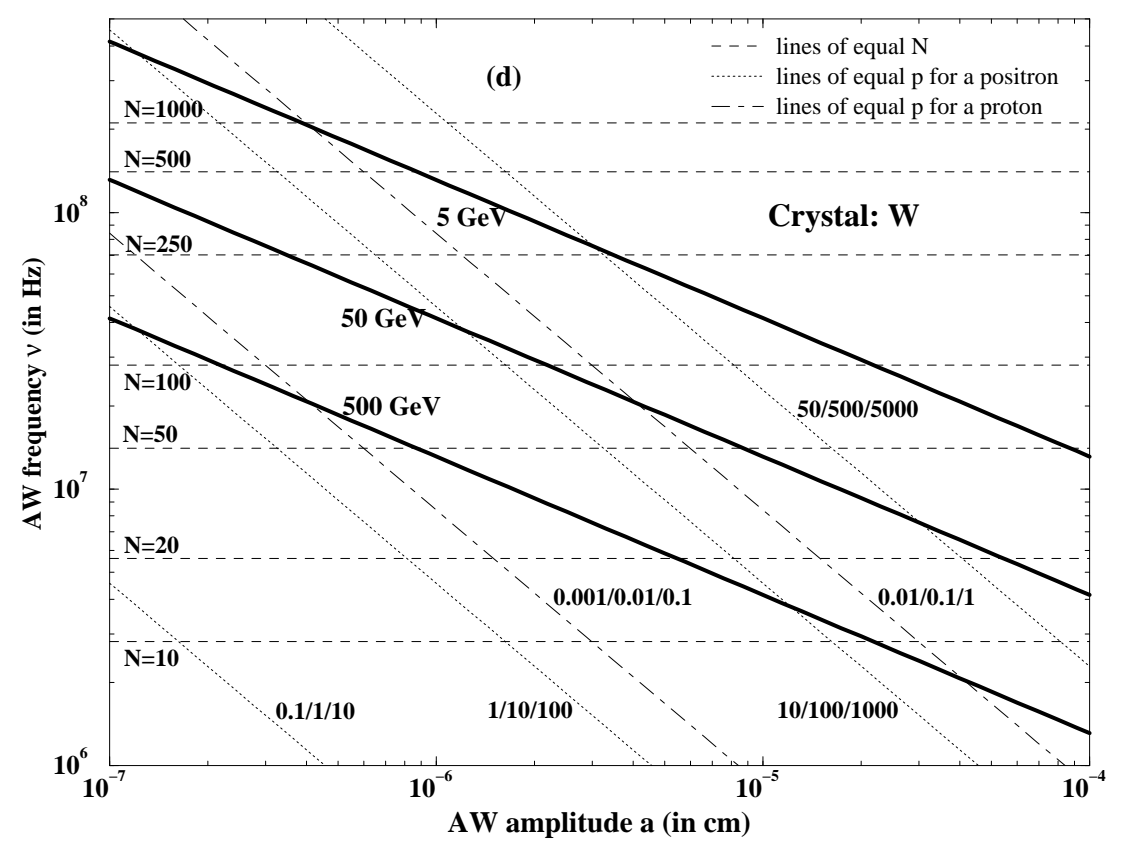

Figure 2. (continued)

\subsection{The undulator conditions}

Now we formulate the conditions (in addition to the channeling condition established above, eqs. (39)-(40)) which allow to consider the system "AW + channeled particle" as an undulator.

Let $L$ denote a crystal thickness. Both the motion of the projectile in the acoustically bent channel and the spectrum of the generated radiation are of the undulator type, only if

$$
\lambda \ll L,
$$

i.e. if the channeling particle oscillates many times within the length of the crystal. As with any other undulator, the suggested undulator is characterized by the frequency, $\omega_{0}$, and the undulator parameter, $p$ (see (20, 23b). These quantities depend on the AW amplitude and the wave length, and on the relativistic factor, and are expressed as follows:

$$
\omega_{0}=c k=2 \pi \frac{c}{\lambda}, \quad p=\gamma \xi \equiv \gamma k a=2 \pi \gamma \frac{a}{\lambda}=2 \pi \gamma \frac{a \nu}{V}
$$

For further reference let us note the difference in functional form of $p$ for the AW undulator and that for the undulators based on magnetic fields (both helical and planar). In the latter case the undulator parameter is equal to (see eg. 47]) $p_{\mathrm{B}}=q B \lambda_{\mathrm{B}} / 2 \pi m c^{2}$, where $B$ is the amplitude value of the magnetic induction and $\lambda_{\mathrm{B}}$ is the period of the magnetic field. The quantity $p_{\mathrm{B}}$ is independent on $\gamma$ whereas $p$ from (42) is linearly proportional to it. 
In figures 2 the dotted and the chain lines indicate the constant values of the undulator parameter $p$. Note that each dotted and chain line corresponds to different values of $p$ depending on the energy of the projectile. Values of $p$ corresponding to the energy $5 / 50 / 500 \mathrm{GeV}$ are shown in figure 2 in the vicinity of each line. The broken lines correspond the constant values of the parameter $N$ both for positron and proton, which is defined as the number of the AW periods per $1 \mathrm{~cm}: N\left(\mathrm{~cm}^{-1}\right)=\lambda^{-1}$. Figures 2 demonstrate that, within the range of validity of the channeling condition (40), the parameters $p$ and $N$ vary over wide ranges: $N=1 \ldots 100, p=0.1-500$ for a positron projectile and $p=0.001-0.1$ for a proton. The upper limiting values of the $p$ 's are larger by more than an order of magnitude than those accessible in the undulators based on the motion of charged particles in periodic magnetic fields 39.

The undulator parameter $p$ is proportional to $a$ (see (42)). In this connection the question arises of the validity of the assumption that $a=$ const during the time of flight, $\tau=L / c$, of the particle through the crystal. Hoevwr, for a running or standing AW the amplitude is time dependent. To formulate a quantitative criterion let us consider the crystal bending by a standing AW. In this case the constant value $a$ must be substituted with $\tilde{a}(t)=a_{o} \cos (2 \pi \nu t)$. For time scales $\tau \ll 1 / \nu$, one gets the following relation for the deviation, $\Delta \tilde{a}=a_{o}-\tilde{a}(t)$, of $\tilde{a}(t)$ from its amplitude on the timescale $\tau: \Delta \tilde{a} / a_{o}=2 \pi^{2}(\tau \nu)^{2}$. Thus, thie time dependence of the AW is negligible provided

$$
\frac{2 \pi^{2}}{c^{2}}(L \nu)^{2}=2 \pi^{2}\left(\frac{V}{c}\right)^{2}\left(\frac{L}{\lambda}\right)^{2} \ll 1
$$

This condition allows to disregard the variations in the shape (3) of the channel on the timescale $\tau$.

To estimate the corresponding values of $L$ let us consider $\nu \leq 100 \mathrm{MHz}$ (see figures(2). Then one gets $L \ll 70 \mathrm{~cm}$ which is more than well-fulfilled for any realistic $L$-value.

Finally, let us demonstrate that the characteristic frequencies of the undulator radiation due to the particle motion along the trajectory (3) and those of the ordinary channeling radiation are well separated, provided conditions (11) and (40) are fulfilled,

The ordinary channeling radiation is due to the transverse oscillations (the first term in (36)) of the particle inside the channel under the action of the interplanar force. The characteristic frequencies of this radiation may be estimated as [32]: $\omega_{\mathrm{c}} \sim \gamma^{2} \chi c$, where $\chi=\left(q U_{\max }^{\prime} / \varepsilon d\right)^{1 / 2}$ (see $(36)$ and $(39)$ ).

The characteristic frequencies of the radiation formed in the acoustically based undulator are given by $\omega_{\mathrm{u}} \sim \gamma^{2} k c=\gamma^{2} \omega_{0}$ (see (42)). Then, the ratio $\omega_{\mathrm{u}} / \omega_{\mathrm{c}}$ is equal to

$$
\frac{\omega_{\mathrm{u}}}{\omega_{\mathrm{c}}} \sim\left(\frac{\varepsilon}{R_{\min } q U_{\max }^{\prime}}\right)^{1 / 2}\left(\frac{d}{a}\right)^{1 / 2} \ll 1,
$$

provided the conditions (11) and (39) are fulfilled. Indeed, for $a \sim 10 d$ and $R_{\min } \sim$ $10 \varepsilon / q U_{\max }^{\prime}$ one gets $\omega_{\mathrm{u}} / \omega_{\mathrm{c}} \sim 10^{-1}$. 
This condition allows to distinguish two mechanisms for the radiation formed in the channeling process in an acoustically bent crystal, and to consider all the radiation in the spectral region $\omega \ll \omega_{\mathrm{c}}$ as formed via the undulator mechanism.

We note here, that the inequality (44) is fulfilled in the whole range of the AW parameters consistent with the channeling condition (40), only if $d<a$ (eq. (11)) is valid too. In the opposite case, $a<d$ [5, 6, 7], which can be realized in the undulators based on a superlattice [6] or/and on a low-amplitude AW [5, 6, 7] the frequencies $\omega_{\mathrm{u}}$ and $\omega_{c}$ can be of the same order of magnitude and, thus, two mechanisms of the radiation formation must be treated simultaneously. As mentioned several times already, in the present paper only the case $d<a$ is considered.

When discussing the motion of the particle in a bent channel and the undulator conditions, we ignored so far the effects of dechanneling and the photon attenuation. The influence of these effects on the undulator radiation will be discussed in the next sections.

Let us summurize our results so far: Provided conditions (1), 40, 41, 43) are fulfilled, the system "ultrarelativistic charged particle + acoustically bent crystal" represents a new type of planar undulator, and, consequently, serves as a new source for highfrequency undulator radiation of high intensity, monochromaticity and a particular pattern of the angular-frequency distribution. The latter as it will be illustrated in the next sections. The validity of the classical treatment of the particle motion in a bent channel allows to apply the quasi-classical approach, described in the previous section, for calculating the characteristics of the undulator radiation.

\section{Numerical results for $\mathrm{d} E(\mathbf{n}) / \mathrm{d} \omega \mathrm{d} \Omega_{\mathbf{n}}$ and $\mathrm{d} E / \mathrm{d} \omega$}

In this section we present numerical results for the spectral and the angular distrbutions of the spontaneous undulator radiation formed during a passage of an ultra-relativistic particle through an acoustically bent crystal. The calculations are performed by using the formalism outlined in section 2. We resctrict ourselves to the case of positron planar channeling.

\subsection{The angular distribution of the radiation}

We start with the illustration of the general features of the angular distribution of the radiation formed in an acoustically based undulator.

Figures 3 and 4 correspond to the case of a $50 \mathrm{GeV}$ positron channeling along (110) plane in a carbon crystal of thickness $L=1 \mathrm{~cm}$. For chosen values of the AW amplitude $a=2.35 \cdot 10^{-7} \mathrm{~cm}$ and frequency $\nu=20 \mathrm{MHz}$, the corresponding magnitudes of the total number of the undulator periods, the undulator parameter and the fundamental harmonic energy are as follows: $N_{\mathrm{u}}=17, p^{2}=10.1, \hbar \omega_{1}=9.9 \mathrm{MeV}$. In a diamond 
crystal the spacing for the (110) planes is $d=1.26 \cdot 10^{-8}$; therefore the condition (四) is well fulfilled.

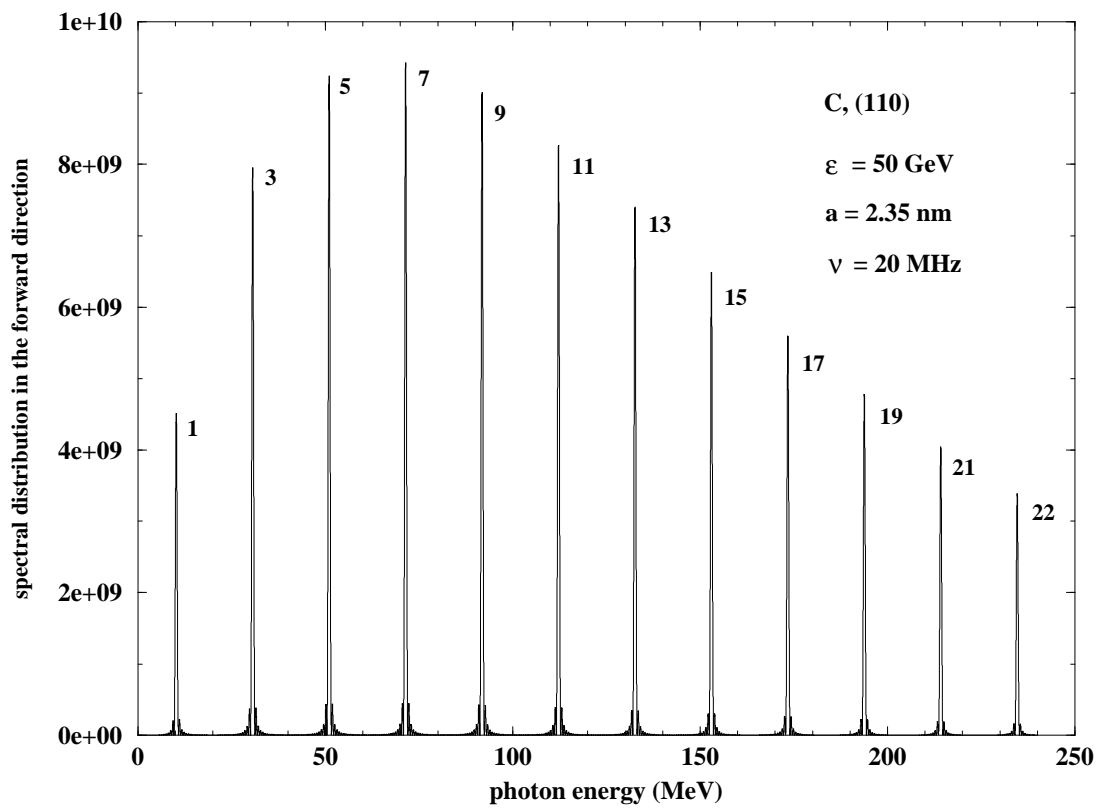

Figure 3. The spectral dependence of the radiated energy for a $50 \mathrm{GeV}$ positron channeling in a diamond. The polar angle of the emission $\theta=0^{\circ}$. Other parameters are as indicated. See also the explanations in the text.

Figure 3 represent the spectral distribution of the radiation emitted along the undulator axis, $\hbar^{-1}\left(\mathrm{~d} E(\mathbf{n}) / \mathrm{d} \omega \mathrm{d} \Omega_{\mathbf{n}}\right)_{\theta=0^{\circ}}$ (see (24)). Each peak corresponds to the emission into the odd harmonics [30, 31, 36], the energies of which are deduced from the relation

$$
\omega_{K}^{\prime}=\frac{4 \gamma^{2} \omega_{0} K}{p^{2}+2}
$$

which is obtained from (13) and (15) by putting $\theta=0^{\circ}$.

It is seen that all harmonics are well separated: the distance between two neighbouring peaks is $2 \hbar \omega_{1} \approx 20 \mathrm{MeV}$ whilst the width of each peak is $\hbar \omega_{1} / N_{\mathrm{u}} \approx 1.2$ $\mathrm{MeV}$. In the vicinity of each main maximum the shape of the spectral distribution is governed by the behaviour of the function $D(\eta)$ defined in (11).

Two 3D plots in figures 4 (a) and 4 (b) allow to visualize the spatial behaviour of the angular distribution of the radiation (at fixed frequency) as a function of the azimuthal, $\varphi$, and the polar, $\theta$, angles of the photon emission. In these figures the quantity $\lg \left(\mathrm{d} E(\mathbf{n}) / \mathrm{d}(\hbar \omega) \mathrm{d} \Omega_{\mathbf{n}}\right)$ (measured along the $z$-axis) as a function of the azimuthal, $\varphi$, and the polar, $\theta$, angles of the photon emission is plotted for two central harmonics. Figure $1(\mathrm{a})$ represents the distribution of the radiation in the odd harmonic $\hbar \omega_{K} \approx 71.4$ 


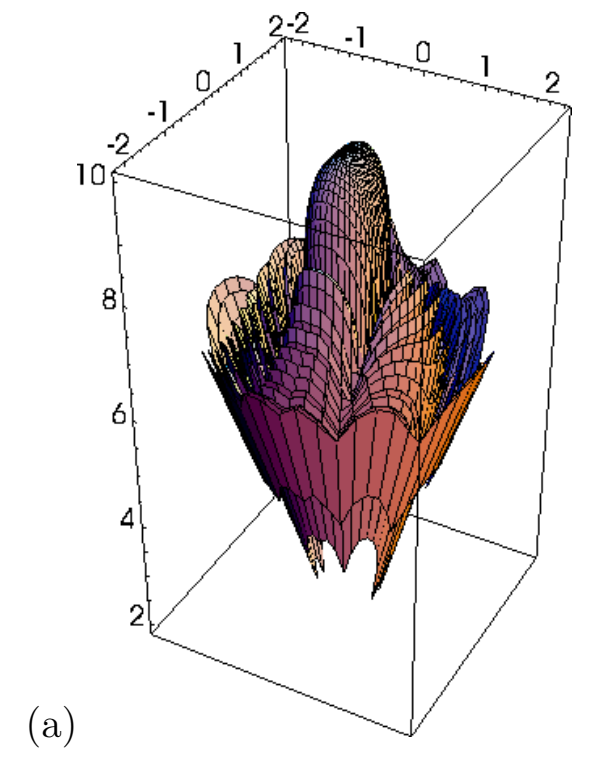

(a)

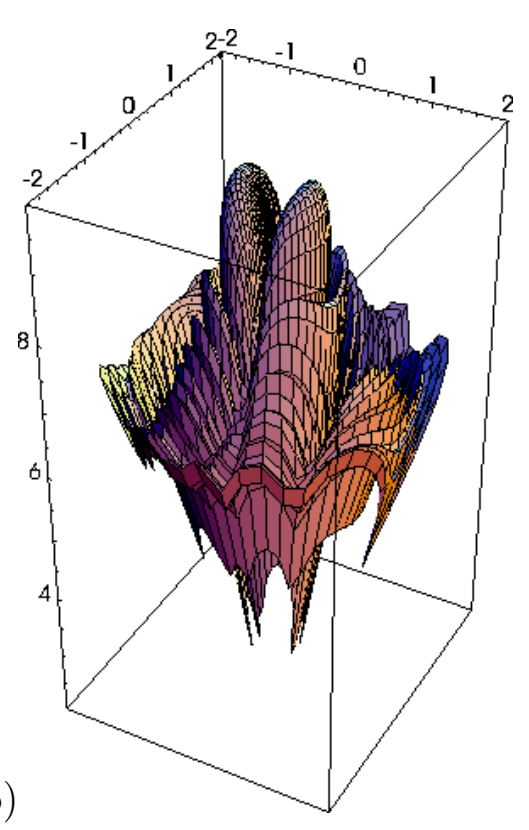

Figure 4. The spatial distribution of the radiation emitted in: (a) the odd harmonic with $K=7$, (b) the even harmonic with $K=6$. Other parameters are as in figure 3 . The undulator axis lies along the $z$ direction. The undulator plane is $y z$. The $x$ and $y$ axis are scaled with respect to the dimensionles parameter $\theta / \theta_{0}$. See also the explanations in the text. 
$\mathrm{MeV}$ with $K=7$, figure $\mathbb{t}(\mathrm{b})$ corresponds to the emission in the even harmonic $\hbar \omega_{K} \approx 61.2 \mathrm{MeV}, K=6$.

The undulator axis lies along the $z$ direction, and $y z$ is the undulator plane. The positive $y$ direction corresponds to $\varphi=0^{\circ}$. In these figures the dimensionless variable $\theta / \theta_{0}$ is used to characterize the distribution in of the radiation with respect to the polar angle. In the considered case the quantity $\theta_{0}$, defined by (21), is equal to $\theta_{0}=23 \mu \mathrm{rad}$.

Figures $6(a, b)$ illustrate general features intrinsic to the planar undulator radiation in the case $p^{2} \gg 1$. We first note that the intensity of the radiation in the odd harmonic is governed by a powerful maximum in the forward direction, whereas there is no radiation in even harmonics for $\theta=0^{\circ}$ The latter reaches its maximal values in nearly forward, but not in forward, directions. Apart from the intensity peak in the forward (for $K$ odd) or nearly forward ( $K$ even) direction, the radiation in a particular harmonic is emitted in a wide range of the polar angles. In both of the figures there are several clearly distinct non-forward peaks in which the radiation intensity reaches the maxima, although the magnitudes of $\mathrm{d} E(\mathbf{n}) / \mathrm{d}(\hbar \omega) \mathrm{d} \Omega_{\mathbf{n}}$ in the maxima at $\theta \neq 0^{\circ}$ rapidly decrease with the polar angle (recall the log scale along the $z$-axis).

Another feature to be mentioned is the abscence of the axial symmetry in the angular distribution shape. More specifically, the radiation emitted within the undulator plane is concentrated in the cone $\theta_{\|} \sim \theta_{0}=p /(\sqrt{2} \gamma)$, whereas the cone angle for the photon emission in the direction perpendicular to the $y z$ plane equals $\theta_{\perp} \sim 1 / \gamma$. Thus, the ratio $\theta_{\|} / \theta_{\perp} \sim p / \sqrt{2}$ characterizes the above mentioned asymmetry in the angular distribution with respect to the atimuthal angle $\varphi$. This ratio is equal to 2.2 in the considered case. This peculiarity most clearly exhibits itself in the shape of the spatial distribution of the radiation in the odd harmonics in the forward direction, see figure 4 (a).

By changing the parameters of the acoustically based undulator (which are the AW amplitude and frequency, the type of a crystal, and the relativistic factor $\gamma$ of the projectile positron) it is possible to vary the intensity of the radiation, the energy, width and the number of emitted harmonics. To illustrate this statement in figures 5(a,b,c) we present the dependencies of the spectral distribution of the radiation emitted at $\theta=0^{\circ}$ for a 2, 5 and $10 \mathrm{GeV}$ positron channelling along (110) planes in Ge and Si crystals. In addition to the parameters indicated in the figures there are other ones, i.e. the crystal length, the number of the undulator periods, the undulator parameter, the fundamental harmonic energy and the width of the line. They are the following:

Figure 5(a): $L=0.1 \mathrm{~cm}, N_{\mathrm{u}}=36, p^{2}=3.25, \hbar \omega_{1}=53 \mathrm{keV}, \hbar \Delta \omega=2.9 \mathrm{keV}$;

Figure 5(b): $L=1 \mathrm{~cm}, N_{\mathrm{u}}=43, p^{2}=6.9, \hbar \omega_{1}=0.23 \mathrm{MeV}, \hbar \Delta \omega=10.5 \mathrm{keV}$;

Figure 5(c): $L=1 \mathrm{~cm}, N_{\mathrm{u}}=43, p^{2}=27.8, \hbar \omega_{1}=0.27 \mathrm{MeV}, \hbar \Delta \omega=12.7 \mathrm{keV}$. 


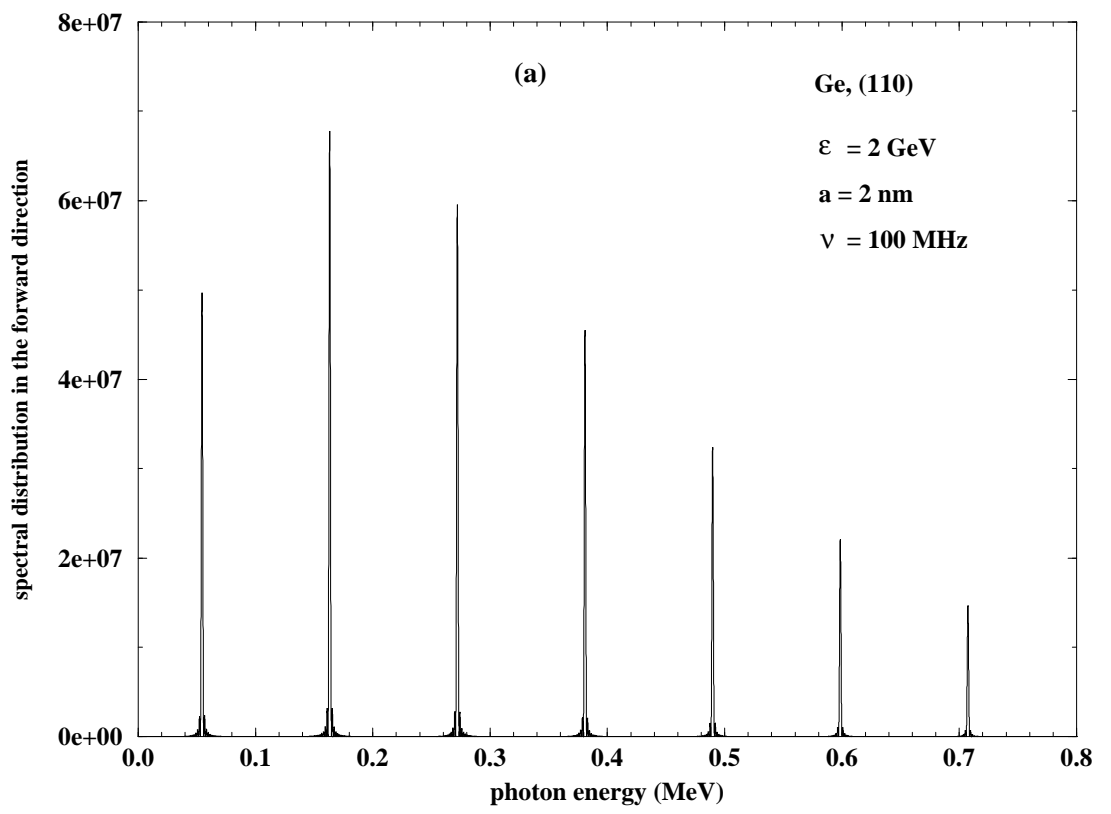

Figure 5. The dependence of $\hbar^{-1} \mathrm{~d} E(\mathbf{n}) / \mathrm{d} \omega \mathrm{d} \Omega_{\mathbf{n}}$ at $\theta=0^{\circ}$ on the photon energy $\hbar \omega$ calculated for a positron channeling in an acoustically bent crystals. The types of the channels, the positron energies and the AW parameters are as indicated. Other parameters are enlisted in the text.

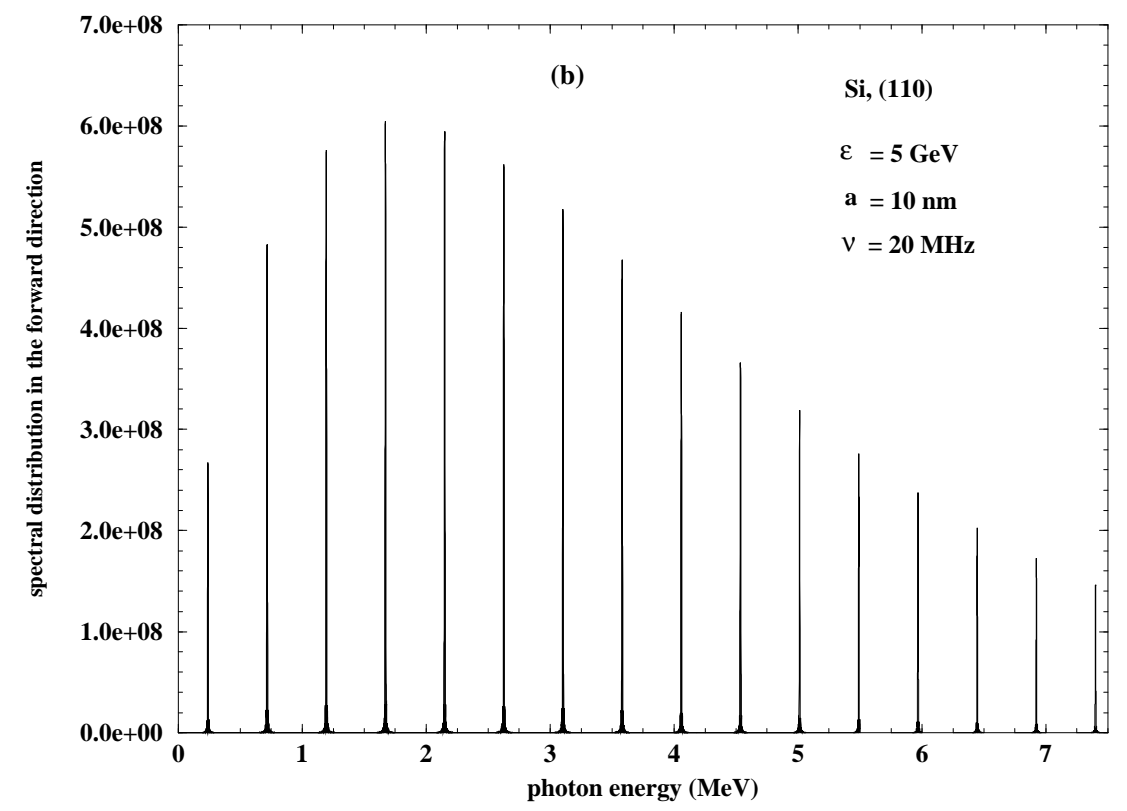

Figure 5. (continued) 


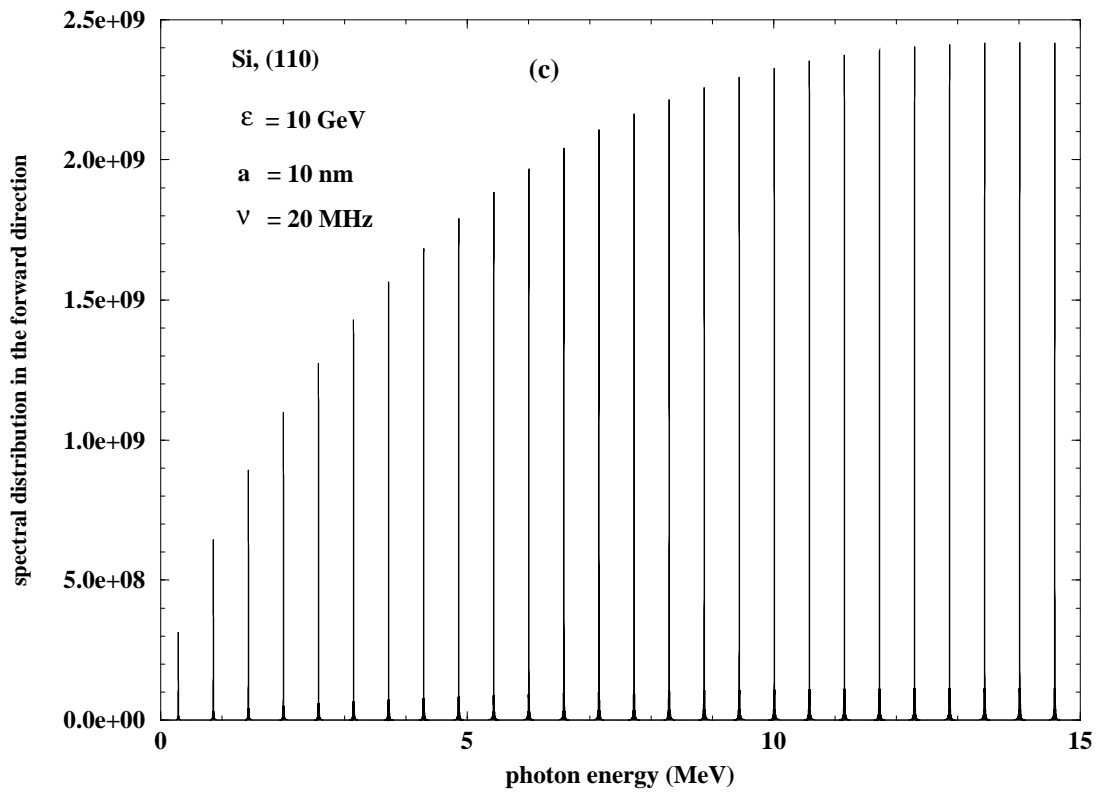

Figure 5. (continued)

\subsection{The spectral distribution of the radiation}

For large undulator parameters, $p>1$, the spectral distribution of the radiation emitted by a charged projectile channeling in a crystal bent by an acoustic wave is obtained from eqs. (26,27). Using these formulae, we calculated the spectral distributions for different parameters of the AW and for various crystals. The results of the calculations are presented in figures 6 -8.

Figures 6(a,b) exhibit dependencies of $\mathrm{d} E / \mathrm{d}(\hbar \omega) / L / N_{u}\left(\right.$ in $\left._{\mathrm{cm}}{ }^{-1}\right)$ for $\varepsilon=50$ and $\varepsilon=500 \mathrm{GeV}$ positrons moving along the (110) channels in a diamond and a tungsten crystal. The AW frequency is fixed at $\nu=10 \mathrm{MHz}$. In these figures each curve shows the $\omega$-dependence obtained at a particular value of the AW amplitude and the corresponding value of the undulator parameter $p$, as indicated. The lowest values

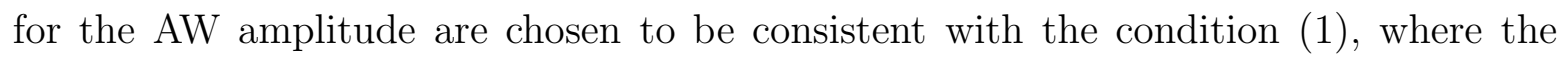
parameter $d$ is equal to $1.26 \AA$ for a diamond and $2.24 \AA$ for a tungsten (110) channels [25]. The largest $a$-values correspond to the relation $\varepsilon /\left(R_{\min } e U_{\max }^{\prime}\right) \approx 1 / 6($ see (39) $)$.

These figures demonstrate that at a certain frequency $\nu$ the intensity of radiation can be varied in a wide range by altering the AW amplitude, which is proportional to the value of the undulator parameter as $a=p /(\gamma k)$, or by choosing the type of a crystal. The latter option affects the value of the undulator parameter via its dependence on the sound velocity $V: p=\gamma(2 \pi \nu / V) a$. Therefore, for equal $a, \nu$ and $\gamma$ the AIR intensity, being proportional to $p$ (see $(26)$ ), is approximately $\left(V_{C} / V_{W}\right) \approx 4$ times higher for a tungsten crystal as compared with that for a diamond.

The radiation intensity can also be varied by changing the AW frequency at 


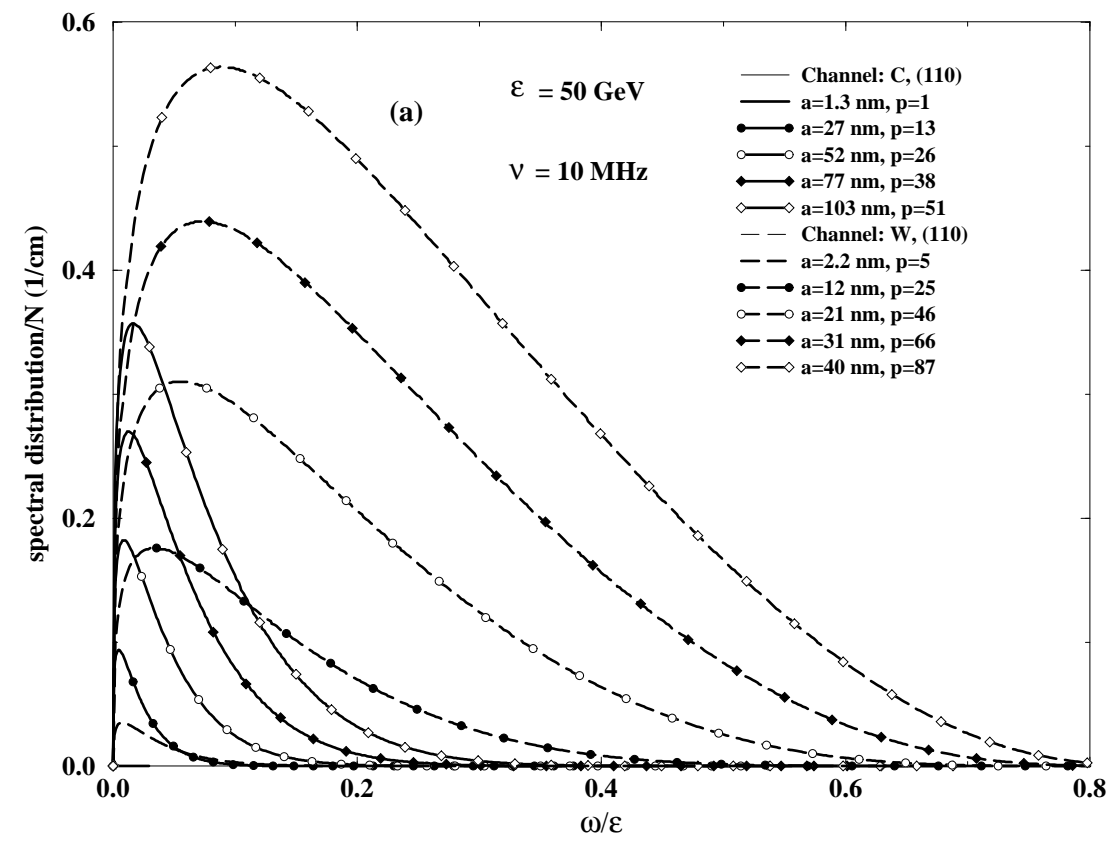

Figure 6. The spectral intensity (per one period) of the AIR, $\mathrm{d} E / \mathrm{d}(\hbar \omega) / L / N_{u}$, emitted by: (a) a $50 \mathrm{GeV}$ positron, (b) a $500 \mathrm{GeV}$ positron, channeling along the (110) plane in $C$ and $W$ crystals calculated for the fixed AW frequency $\nu=10 \mathrm{MHz}$ and for various values of the AW amplitude $a$ and the corresponding undulator parameters $p$ (as indicated). Further explanations are given in the text.

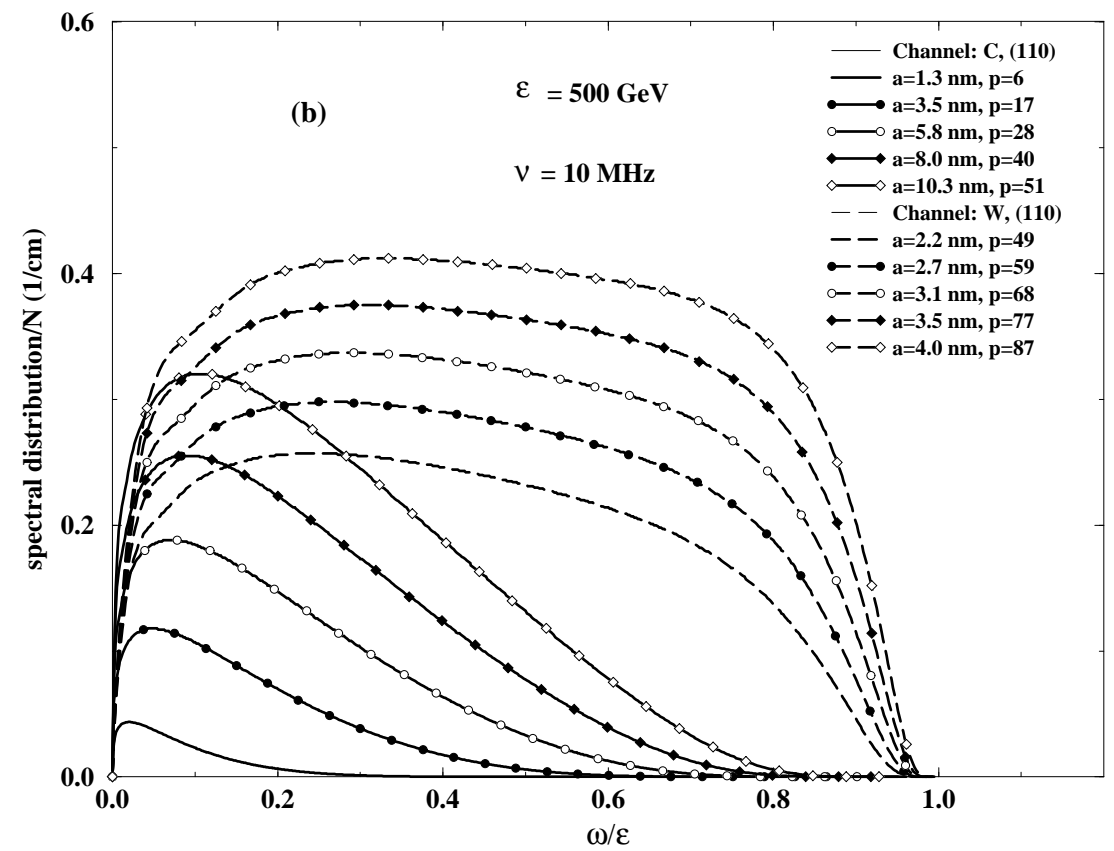

Figure 6. (continued) 


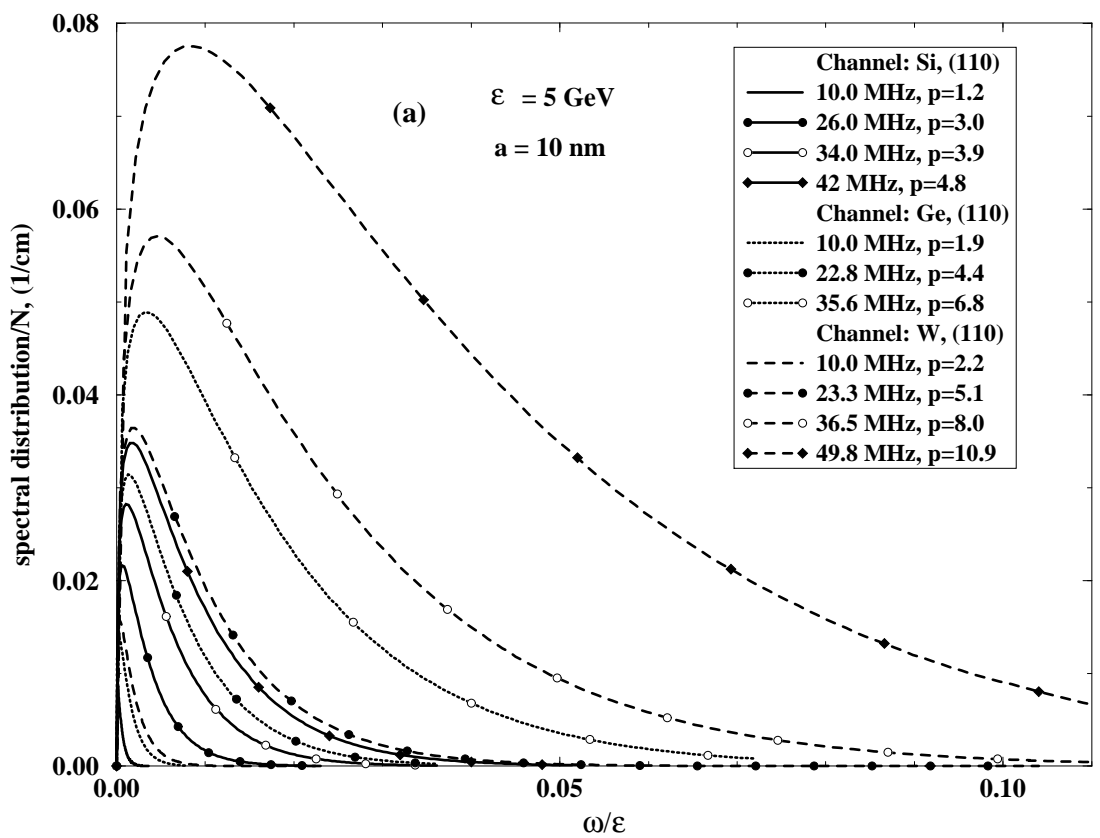

Figure 7. The spectral intensity (per one period) of the AIR, $\mathrm{d} E / \mathrm{d}(\hbar \omega) / L / N_{u}$, emitted by: (a) a $5 \mathrm{GeV}$ positron, (b) a $50 \mathrm{GeV}$ positron, channeling along the (110) plane in $S i, G e$ and $W$ crystals calculated for the fixed AW amplitude $a=10 \mathrm{~nm}$ and for various values of the AW frequency $\nu$ and the corresponding undulator parameters $p$ (as indicated). See also explanations in the text.

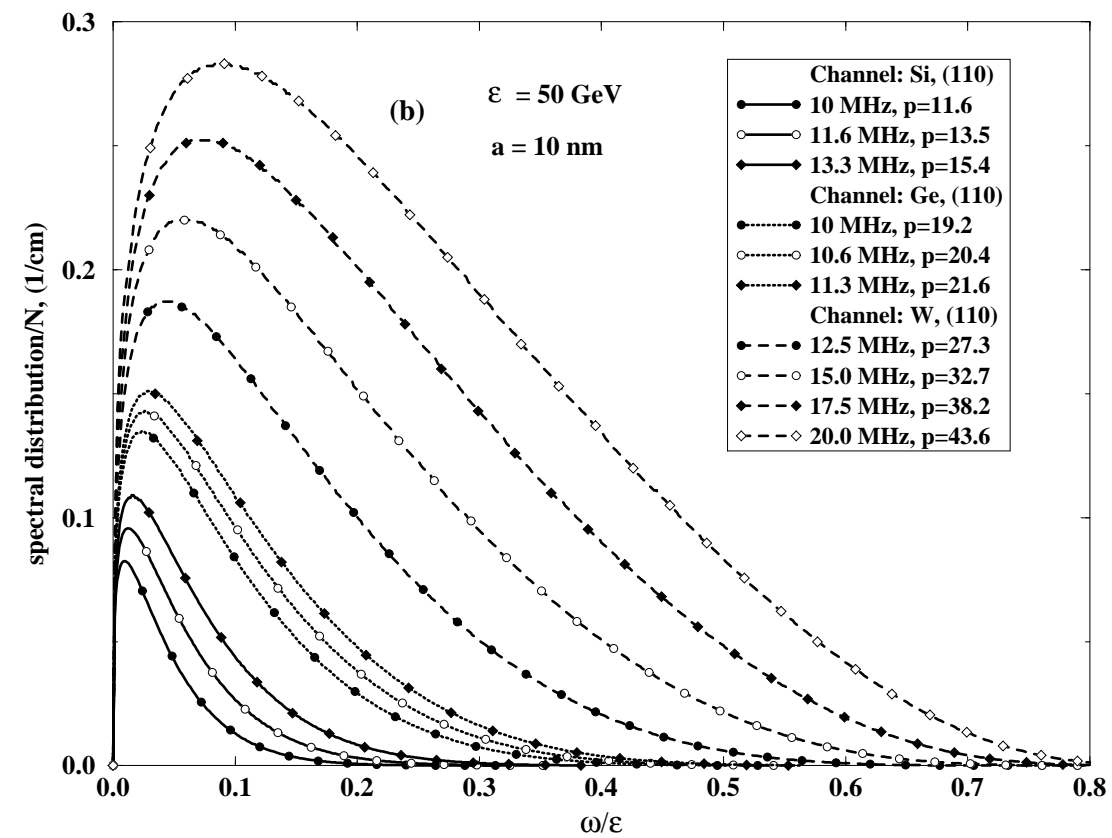

Figure 7. (continued) 


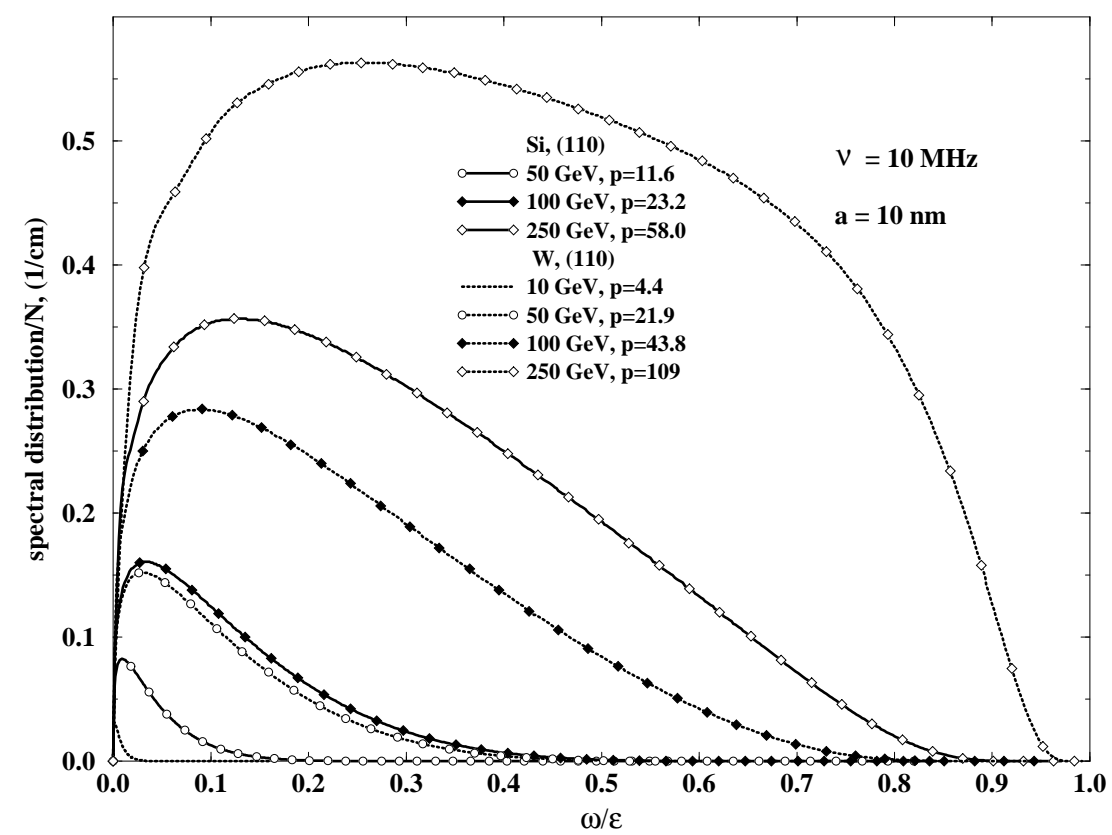

Figure 8. The spectral intensity (per one period) of the AIR, $\mathrm{d} E / \mathrm{d}(\hbar \omega) / L / N_{u}$, emitted by a positron of various $\varepsilon$ (as indicated) channeling along the (110) plane in Si and $W$ crystals calculated for the fixed AW amplitude $a=10 \mathrm{~nm}$ and frequency $\nu=10 \mathrm{MHz}$. The corresponding undulator parameters $p$ are indicated.

some constant value of $a$. This possibility is illustrated by figures 7(a,b) where the dependencies of $\mathrm{d} E / \mathrm{d}(\hbar \omega) / L / N_{u}$ on $\omega$ for $\varepsilon=5$ and $\varepsilon=50 \mathrm{GeV}$ positron channeled in Si, Ge and $W$ crystals are presented.

The spectral distributions calculated at different energies $\varepsilon$ of a positron channeling in $S i$ and $W$ crystals are compared in figure 8 .

The features to be discussed in connection with all these figures are the pattern of the spectral distribution shape and its transformation with the increase in $\varepsilon$. It was noted above (see the discussion below (24)) that for $\omega^{\prime} \gg \omega_{\max }^{\prime} \approx p \gamma^{2} \omega_{0}$ the intensity of the undulator radiation (both the angular and the spectral ones) exponentially decreases to zero. Within the frame of the quasi-classical approximation the photon frequency $\omega$ is related to the quantity $\omega^{\prime}$ through the first equality in (6). Thus, the intensity of radiation effectively falls off for the photon energies satisfying the relation

$$
\frac{\hbar \omega}{\varepsilon}>\frac{1}{1+\varepsilon / p \gamma^{2} \hbar \omega_{0}}=\frac{1}{1+a / \lambda_{c} p^{2}}
$$

Here $\lambda_{c}=\hbar / m c=2.42 \cdot 10^{-10} \mathrm{~cm}$ is the Compton wavelength of the positron. To obtain the equality in (46) we made use of $p=\gamma k a$ and $\omega_{0}=k c$.

In the case of $\left(a / \lambda_{c}\right) p^{-2} \gg 1$, which is achieved by choosing relatively low values of the parameters $\gamma, a$ or $\nu$, the spectrum of radiation is concentrated in the region of low photon energies, $\hbar \omega / \varepsilon \ll 1$ whereas for $\hbar \omega / \varepsilon \leq 1$ it rapidly drops to zero, as follows 
from the estimation (46). Such a behaviour most vividly is illustrated by figure 7(a) (see also the curves for the C (110) channel in figure 6(b) and for the Si (110) and Ge (110) channels in figure 7(b)).

By increasing $\gamma$ the parameter $\left(a / \lambda_{c}\right) p^{-2}$ becomes $<1$, leading to a prominent increase in the intensity of radiation in the high-energy photon region, $\hbar \omega / \varepsilon \leq 1$. The curves for the $W$ (110) channel in figure $6(\mathrm{~b})$ and the curve for a $250 \mathrm{GeV}$ positron channelling in W (110) (see figure 8) clearly exhibit the transformation of the spectral distribution shape: it becomes flatter, thus reflecting that, except for the regions $\hbar \omega / \varepsilon \longrightarrow 0$ and $\hbar \omega / \varepsilon \longrightarrow 1$, the probability of the photon emission is almost independent on the photon energy.

\subsection{The energy loss due to the AIR}

The stability of spontaneous undulator radiation formed during the passage of the projectile through an acoustically bent crystal of total length $L$ to a great extend is subject to the condition $\Delta \varepsilon / \varepsilon \ll 1$, where $\Delta \varepsilon$ is the absolute decrease in the projectile energy, $\varepsilon$, due to various inelastic processes. In particular, the above-written inequality implies, that the energy loss, $\Delta \varepsilon$, due to spontaneous AIR only must be small compared with $\varepsilon$.

To estimate this quantity in the case $p^{2} \gg 1$ it is necessary to integrate $\mathrm{d} E / \mathrm{d} \omega$ from (26) over the interval $\hbar \omega=0 \ldots \varepsilon$. On the other hand, to obtain $\Delta \varepsilon$ for $p^{2}<1$ it is sufficient to use the classical expression (28) for the spectral and angular distribution of the radiation and to carry out the integrations over the angles $\varphi$ and $\theta$ and over the photon energies.

In figures $9(\mathrm{a}, \mathrm{b})$ the dependencies of the relative energy loss, $\Delta \varepsilon / \varepsilon$, on the energy of the projectile $\varepsilon$ is plotted for a positron channeling in (110) channels for $C, S i$ and $W$ crystals and for various AW frequencies as indicated. The curves in figure 9(a) correspond to the AW amplitude $a=2 \mathrm{~nm}$, which is equal, by the order of magnitude, to $10 d$ for all crystals considered. In figure 9(b) $a=10 \mathrm{~nm}$. For all curves in these figures the AW frequency and amplitude are subject to the strong inequality $\nu^{2} a \ll C$ (see eq. (40)). Both figures correspond to a crystal length $L=1 \mathrm{~cm}$. The total number of the undulator periods, $N_{\mathrm{u}}$ is related to $L, \nu$ and the AW velocity $V$ according to $N_{\mathrm{u}}=L \cdot V / \nu$.

Except for the case $p^{2}<1$, it is hardly feasible to establish by explicit analytical means the ranges of parameters inside which the inequality $\Delta \varepsilon / \varepsilon \ll 1$ is valid. Indeed, expressions (26) and (27) exhibit quite complicated dependence of $\mathrm{d} E / \mathrm{d} \omega$, and, consequently, that of $\Delta \varepsilon$ on $\varepsilon, a$ and $\nu$. In addition to these the energy loss is dependent (indirectly, through the conditions (10), (40) and (41)) on the parameters of the crystal, i.e. on $d, U_{\max }^{\prime}$ and $V$ and on its length $L$. 


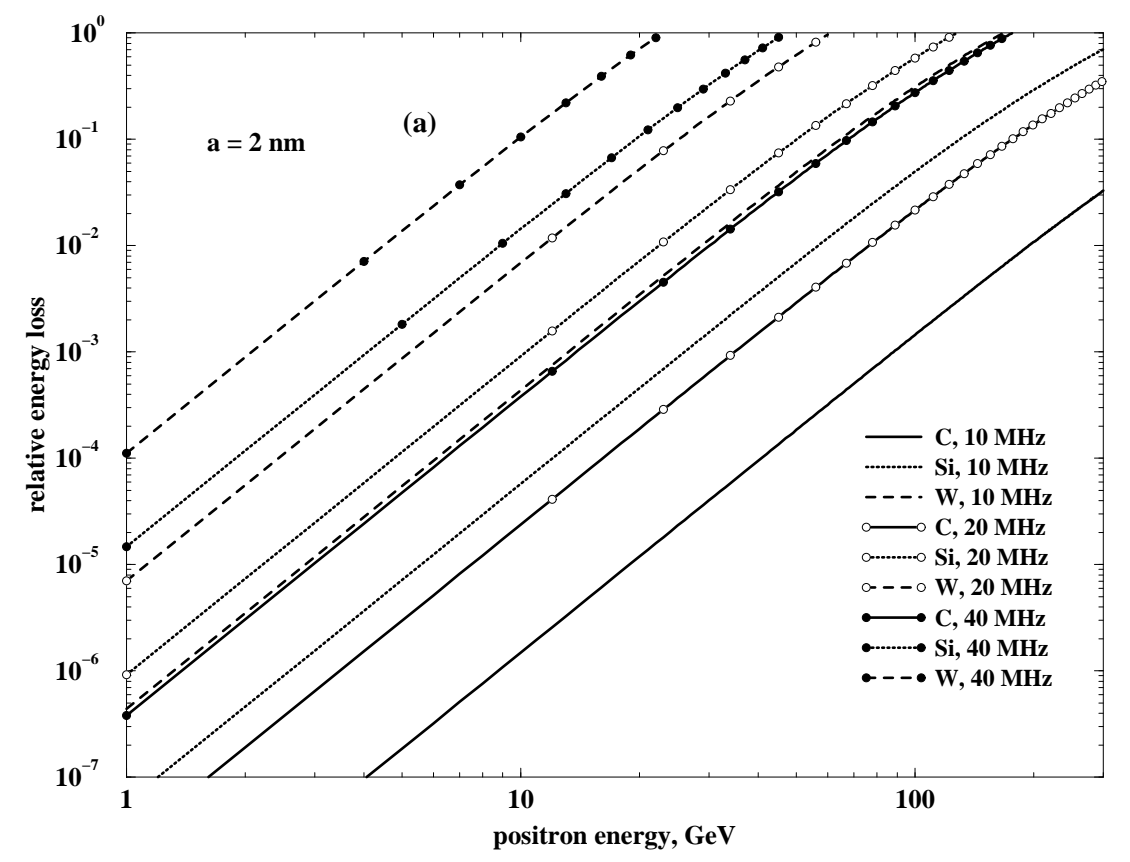

Figure 9. The relative energy loss, $\Delta \varepsilon / \varepsilon$, due to the acoustically induced radiation versus the energy of a positron channeling along the (110) plane in $C, S i$ and $W$ crystals calculated for the fixed AW amplitude: (a) $a=2 \mathrm{~nm}$, (b) $a=10 \mathrm{~nm}$, and for various values of the AW frequency $\nu$ (as indicated). The curves correspond to the crystal length $L=1 \mathrm{~cm}$.

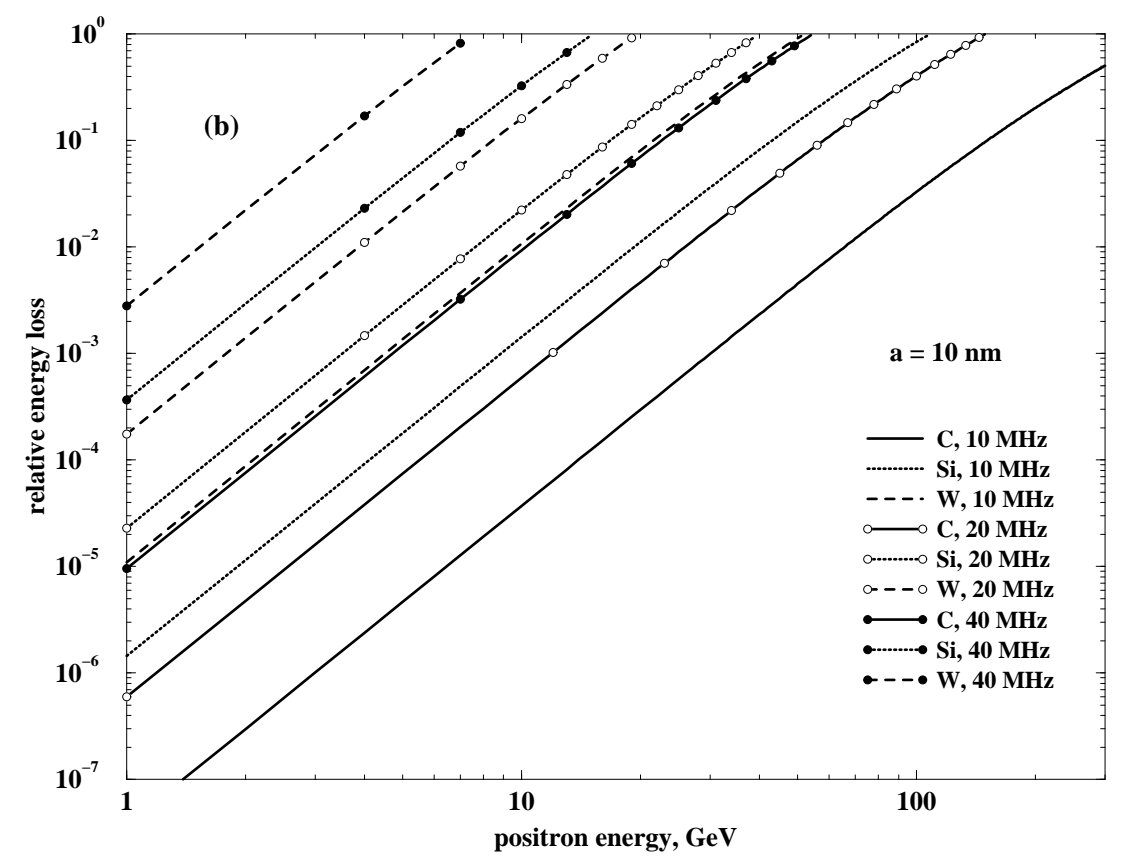

Figure 9. (continued) 
Thus, for any particular set of the above-mentioned parameters, the magnitude of the energy loss must be calculated numerically. Nevertheless, figures 9 allow to estimate the range of validity of $\Delta \varepsilon / \varepsilon \ll 1$. It is seen that for $a=10 \ldots 100 d$ and $L \leq 1 \mathrm{~cm}$ there are wide ranges of positron energy ( $\varepsilon$ from $\leq 1 \mathrm{GeV}$ up to several tens of $\mathrm{GeV}$ ) and AW frequency ( $\nu$ from several $\mathrm{MHz}$ up to several tens of $\mathrm{MHz}$ ) for which all the undulator conditions (40, 41, 43) are fulfilled and, simultaneously, the relative energy loss due to the acoustically induced radiation is negligibly small.

\section{Stimulated emission}

In this section we discuss the possibility to generate stimulated emission of high energy photons by means of a bunch of ultra-relativistic particles of length $L_{\mathrm{b}}$ moving in a channel bent by a transverse acoustic wave.

The mechanism of the emission stimulation is illustrated in figure 10. The photons emitted in the nearly forward direction at some maximum/minimum point of the trajectory by a group of particles of the bunch stimulate the emission of the same photons by another (succeding) group of particles of the same bunch when it reaches next maximum/minimum.

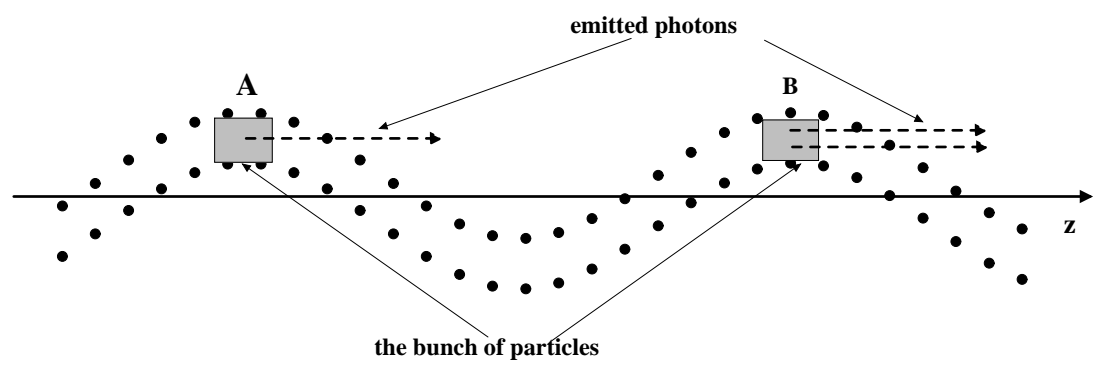

Figure 10. Mechanism of the radiation stimulation in the AW based undulator. Photons, which are emitted in the forward direction by the positron bunch (dark block) as it passes the undulator maximum (marked with A), stimulate the emission by the same bunch in the vicinity of the next maximum (marked with B). It is assumed that the length of the bunch, $L_{b}$, its velocity, $v$, and the undulator period $\lambda$, satisfy the condition $(1-v / c) \lambda \ll L_{b}$, which means that the photon slippage against the positron bunch during one undulator period is much less than the latter.

This scheme implies that the stimulation is due to the motion of the same bunch along the trajectory (3). Therefore, the bunch velocity $v$, its length $L_{\mathrm{b}}$ and the length of the crystal $L$ must satisfy the condition

$$
\frac{L}{c} \geq \frac{L-L_{\mathrm{b}}}{v}, \quad \text { or } \quad \frac{v}{c} \geq 1-\frac{L_{\mathrm{b}}}{L}
$$

which means that the electromagnetic wave does not slip against the bunch of particles 
by more than $L_{\mathrm{b}}$ on the lenghtscale $L \gg L_{\mathrm{b}}$ and, thus, the amplification occurs during each of the undulator periods of total number $N_{\mathrm{u}}=L / \lambda$.

In the theory of free-electron lasers (FEL) this principle is called "Self-Amplified Spontaneous Emission (SASE)" [48, 19] and usually is referred to the FEL operation in the high gain (or collective instability) regime [50, 51, 52, 53].

An accurate treatment of the evolution of the photon pulse in the SASE mode implies that the space charge fields, energy spread of the particles in the beam and diffraction effects are taken into account [53]. In the case when the beam undergoes channeling the picture must be additionally complicated by considering the dechanneling dynamics [25] as well as the photon attenuation [54].

We do not aim to fulfil this program in the present paper. Instead, for the suggested undulator, we shall present an estimate of the gain exploiting the general relationship between gain and spontaneous spectrum of undulator radiation [30, 55]. Thus, we disregard the dynamics of the particles in the undulator and consider the case of a monoenergetic beam of independent particles. Additionally we assume that there is no beam divergence. The effects of dechanneling and photon attenuation are discussed qualitatively on a phenomenological level.

\subsection{General expression for the gain}

It is our aim to establish the range of parameters (which are: the AW amplitude and frequency (wavelength), the type of the particle and its energy, and the type of a crystal) within which there is a principle possibility to consider the SASE radiation by means of the acoustically bent crystal.

It had been established long ago [56] that in a free-electron device the stimulation of emission occurs if only the photon frequency is equal to that of the spontaneous undulator radiation, $\omega_{K}$ (see (13)).

Let us estimate the gain factor, $g_{K}$, which defines the increase (i.e. the case $g_{K}>0$ ) or the decrease (if $g_{K}<0$ ) per $1 \mathrm{~cm}$ in the total number, $N_{K}$, of the emitted photons at a frequency $\omega_{K}$

$$
\mathrm{d} N_{K}=g_{K} N_{K} \mathrm{~d} z,
$$

due to stimulated emission (absorption) by the particles in the beam. We shall do this within the frame-work of low-gain approximation (see eg. [53]). Also we restrict our consideration to the case of the stimulated emission of soft photons, $\hbar \omega \ll \varepsilon$, and, hence, $\omega^{\prime}=\omega($ see (6) $)$.

The general expression for the quantity $g_{K}$ is

$$
g_{K}=n\left[\sigma_{\mathrm{e}}\left(\varepsilon, \varepsilon-\hbar \omega_{K}\right)-\sigma_{\mathrm{a}}\left(\varepsilon, \varepsilon+\hbar \omega_{K}\right)\right]
$$

Here $\sigma_{\mathrm{e}}\left(\varepsilon, \varepsilon-\hbar \omega_{K}\right)$ and $\sigma_{\mathrm{a}}\left(\varepsilon, \varepsilon+\hbar \omega_{K}\right)$ are the cross sections of, correspondingly, the 
spontaneous emission and absorption of the $\omega_{K}$-photon by a particle of the beam, $n$ stands for the volume density of the beam particles. By using the known relations between the cross section of the photon emission/absorption and the spectral-angular intensity of the emitted radiation [42], and taking into account the relation $\hbar \omega \ll \varepsilon$, one gets the following expression for the gain, corresponding to the increase in the number of photons of the frequency within the interval $\omega_{K} \pm \Delta \omega$ emitted in the cone $\Delta \Omega_{K}$ (see below) with the axis along the the $z$-direction:

$$
g_{K}=-(2 \pi)^{3} \frac{c^{2}}{\omega_{K}^{2}} n \frac{\mathrm{d}}{\mathrm{d} \varepsilon}\left[\frac{\mathrm{d} E}{\mathrm{~d} \omega_{K} \mathrm{~d} \Omega}\right]_{\theta=0} \Delta \omega \Delta \Omega_{K}
$$

The width $\Delta \omega$ one gets from (13) by substituting $\omega^{\prime}$ with $\omega, \Delta \omega=$ $\left(2 / N_{\mathrm{u}}\right)\left(4 \gamma^{2} \omega_{0} /\left(2+p^{2}\right)\right)$.

Making use of (13) (see also (19)) one gets the estimation of the quantity $\Delta \Omega_{K}$ :

$$
\Delta \Omega_{K}=\frac{2 \pi\left(1+2 p^{-2}\right)}{K} \theta_{0}^{2}
$$

It defines the maximal solid angle along the undulator axis inside which the $K$ th harmonic is well resolved. The quantity $\theta_{0}^{2}$ is given in (21). Expression (51) is valid for both $p^{2} \gg 1$ and $p^{2} \ll 1$. In the latter case one may set $\left(1+2 p^{-2}\right) \theta_{0}^{2} \approx \gamma^{-2}$.

For the energy emitted in the forward direction $(\theta=0)$ the formulas (24) and (28) yield:

$$
\begin{aligned}
& {\left[\frac{\mathrm{d} E}{\mathrm{~d} \omega_{1} \mathrm{~d} \Omega}\right]_{\theta=0}=\frac{q^{2}}{c} N_{\mathrm{u}}^{2} \gamma^{2} p^{2} D(\eta), \quad \text { for } p^{2} \ll 1} \\
& {\left[\frac{\mathrm{d} E}{\mathrm{~d} \omega_{K} \mathrm{~d} \Omega}\right]_{\theta=0}=\frac{q^{2}}{c} N_{\mathrm{u}}^{2} \frac{\gamma^{2}}{p^{2}}(2 K)^{2 / 3} D(\eta) \quad \text { for } p^{2} \gg 1}
\end{aligned}
$$

Expression (52d) is denoted for the fundamental harmonic, $\omega_{1}=2 \omega_{0} \gamma^{2}$ in accordance with (28). In (528) we assumed that $K \ll K_{\max } \sim p^{3}$ and took into account that in this case the argument $\zeta$ (see (25)) of the derivative of the Airy function from (24) is small, so that $16 \mathrm{Ai}^{\prime 2}(0) \approx 1[45]$.

When carrying out the derivative of $\left[\mathrm{d} E / \mathrm{d} \omega_{1} \mathrm{~d} \Omega\right]_{\theta=0}$ with respect to $\varepsilon$ the main contribution comes from the term $\mathrm{d} D(\eta) / \mathrm{d} \varepsilon=(\mathrm{d} \eta / \mathrm{d} \varepsilon) \cdot(\mathrm{d} D(\eta) / \mathrm{d} \eta)$. To obtain maximal positive gain it is necessary to choose that slope of $D(\eta)$ where it is negative. Then the extremal value of this derivative is equal to

$$
\begin{array}{ll}
\frac{\mathrm{d} D(\eta)}{\mathrm{d} \varepsilon}=\mathrm{d} \eta / \mathrm{d} \varepsilon \frac{\mathrm{d} D(\eta)}{\mathrm{d} \eta} \approx \frac{-2}{\varepsilon} \cdot \frac{N_{\mathrm{u}}}{2} & \text { for } p^{2} \ll 1 \\
\frac{\mathrm{d} D(\eta)}{\mathrm{d} \varepsilon}=\mathrm{d} \eta / \mathrm{d} \varepsilon \frac{\mathrm{d} D(\eta)}{\mathrm{d} \eta} \approx \frac{-4 K}{\varepsilon p^{2}} \cdot \frac{N_{\mathrm{u}}}{2} & \text { for } p^{2} \gg 1
\end{array}
$$

The first factors on the right-hand sides of (53d) and (53b) correspond to $(\mathrm{d} \eta / \mathrm{d} \varepsilon)$ and, as it is seen, their magnitude depends on $p^{2}$. The last factor $\left(N_{\mathrm{u}} / 2\right)$ equals to the 
maximum value of the derivative $(\mathrm{d} D(\eta) / \mathrm{d} \eta)$ with $\eta$ lying within the interval $K \pm 1 / N_{\mathrm{u}}$. We concentrate our attention on these details in order to point out the nature of the difference in the $p$-dependence of $g_{K}$ for the AW undulator and that for the undulator based on magnetic field. As it was noted above (see the paragraph after(42)) in the latter case the undulator parameter is independent of $\gamma$ resulting in $\mathrm{d} \eta / \mathrm{d} \varepsilon=-2 \mathrm{~K} / \varepsilon$ for both $p_{\mathrm{B}}^{2} \ll 1$ and $p_{\mathrm{B}}^{2} \gg 1$ cases $\left(K=1\right.$ if $\left.p_{\mathrm{B}}^{2} \ll 1\right)$. In turn it leads to the proportionality of the gain to $p_{\mathrm{B}}^{2}$ regardless to the magnitude of the latter [47, 57]. In our case the gain $g_{K}$, which is equal to

$$
\begin{array}{ll}
g_{K}\left(\mathrm{~cm}^{-1}\right) \approx(2 \pi)^{3} \frac{q^{2}}{m} r_{\mathrm{cl}} \frac{N_{\mathrm{u}}^{2} \lambda}{\gamma^{3}} n p^{2} & \text { for } p^{2} \ll 1 \\
g_{K}\left(\mathrm{~cm}^{-1}\right) \approx \frac{2(2 \pi)^{3}}{(2 K)^{4 / 3}} \frac{q^{2}}{m} r_{\mathrm{cl}} \frac{N_{\mathrm{u}}^{2} \lambda}{\gamma^{3}} n & \text { for } p^{2} \gg 1,
\end{array}
$$

is independent on $p$ if $p^{2} \gg 1$. In the above equations $\lambda_{\mathrm{c}}=\hbar / m_{\mathrm{e}} c=3.9 \cdot 10^{-11} \mathrm{~cm}$ is the Compton wavelength and $r_{\mathrm{cl}}=e^{2} / m_{\mathrm{e}} c^{2}=2.8 \cdot 10^{-13} \mathrm{~cm}$ is the classical radius of the electron, $q$ and $m$ are the charge and the mass of the projectile measured in units of the elementary charge $e>0$ and electron mass $m_{\mathrm{e}}$, respectively. The quantity $\lambda$ is measured in $\mathrm{cm}$ and $n$ in $\mathrm{cm}^{-3}$.

Note the strong inverse dependence of $g_{K}$ on $\gamma$ which is due to the radiative recoil. The gain is proportional to the factor $N_{\mathrm{u}}^{2}$, which reflects the coherence of radiation. The proportionality of the gain to $\lambda$ means that the increase in $\lambda$ leads to an enchancement of the radiation intensity in the forward direction.

Expressions (54a) and (54b) were obtained by using the asymptotic formulae (24) and (28) valid for $p^{2} \gg 1$ and $p^{2} \ll 1$, respectively. Extrapolating (54a) and (54b) to the region $p^{2} \sim 1$ and considering in (54b) the fundamental harmonic, $\omega_{1} \approx 4 \gamma^{2} \omega_{0} / p^{2}$, one recognizes that in the point $p=1(54 b)$ is within a factor $2^{-1 / 3}=0.79$ of (54a). Such a discrepancy may be ignored having in mind the approximate character of the initial formulae (24) and (28).

Apart from the absence of the $p^{2}$ dependence in case $p^{2} \ll 1$ (due to the reasons mentioned above) expressions (54d) and (54b) have the same functional form as those obtained for the undulator based on the action of periodic magnetic field [56, 58, 57, 47].

\subsection{Estimations of $g_{K}$ for the $A W$ based undulator}

Expressions (54a) and (54b) allow to make quantitative estimates concerning the possibility of stimulated emission of the high-energy photons.

Before carrying out this analysis let us note two main qualitative features which distinguish the AW based undulator and that based on the action of the periodic magnetic field. In the latter case both the particle and the photon beams propagate in vacuum. Therefore, there are no strong mechanisms leading to the decrease in the 
beam density.

In the scheme considered here both beams propagate in a crystal. Thus, an adequate description of the stimulated emission with necessity must account for perticle dechanneling and for photon attenuation.

Random scattering of the channeling particle by the electrons and nuclei of the crystal leads to the gradual increase of the particle energy associated with the transverse oscillations in the channel. As a result, the transverse energy at some distance, $L_{d}$, from the entrance point exceeds the depth of the interplanar potential well, and the particle leaves the channel. The quantity $L_{d}$ is called the dechanneling length [25].

The dynamics of the photon flux propagation, apart from the stimulation effect, is strongly influenced by a variety of processes occuring in a crystal. These are the atomic and the nuclear photoeffects, the coherent and the incoherent scattering with electrons and nuclei, the electron-positron pair production (in the case of high energy photons). All these processes lead to the decrease in the density of the photon flux as it propagates through the crystal.

The rigorous treatment of the stimulated emission in the system "channelng beam + photon flux", with both the dechanneling effect and the photon attenuation accounted for, must include solving the coupled equations of the Fokker-Planck form for the particle beam [25, 32] and for the photon beam [60]. This will be done at a later time. In this paper we present the quantitative analysis of the stimulated emission by a beam channeling in the AW bent crystal by using phenomenological arguments when treating the dechanneling and the photon attenuation.

Let us first estimate the role of the dechanneling effect.

To simplify the expressions, we will only consider the stimulated emission of the fundamental harmonic

$$
\omega=\frac{4 \omega_{0} \gamma^{2}}{2+p^{2}},
$$

and omit the index " 1 " in the notations $\omega_{1}$ and $g_{1}$. Also, we restrict the treatment to the case of a positron channeling, $M=Z=1$.

By expressing the total number of the undulator periods $N_{\mathrm{u}}$ through the undulator length, $N_{\mathrm{u}}=L / \lambda$, and using (54a) and (54b), one gets the following estimate for $g$ :

$$
g \equiv g(L) \approx \Theta(p)(2 \pi)^{2} r_{\mathrm{cl}} n \frac{k L^{2}}{\gamma^{3}}
$$

where the quantity $\Theta(p)$ is defined as

$$
\Theta(p)= \begin{cases}1 & \text { for } p^{2} \geq 1 \\ p^{2} & \text { for } p^{2}<1\end{cases}
$$

In (57) we have omitted the factor $2^{-1 / 3}=0.79 \approx 1$ in the case $p^{2} \geq 1$. In (56) the 
argument $L$ in the notation $g(L)$ stresses the dependence of the gain factor on the crystal length.

Provided the dechanneling is neglected, one may unrestrictedly increase $g$ by considering larger $L$-values. In reality, the volume density of the channeling particles decreases with the penetration distance, $z$, and, roughly, satisfies the exponential decay law [25]

$$
n(z)=n_{0} \exp \left(-z / L_{d}(\gamma, R)\right)
$$

where $n_{0}$ is the volume density at the entrance, $L_{d}(\gamma, R)$ is the dechanneling length. For given crystal and channel $L_{d}(\gamma, R)$ depends on a positron energy (relativistic factor) and on the curvature radius $R$.

As argued in [25], the decrease of $n$ with $z$ acquires the exponential form (58) at a considerable penetration depth, $z \geq L_{d}(\gamma, R)$. If the crystal length is less than $L_{d}(\gamma, R)$ then the dependence $n(z)$ is strongly influenced by the initial conditions (the incident angle, the beam divergency) of the beam impacting on the crystal. Therefore, for estimation purposes, we set the length of the crystal equal to the dechanneling length, $L=L_{d}(\gamma, R)$, and assume that the beam density for $z<L$ is equal to its initial value $n_{0}$.

For a bent crystal with a constant curvature radius $R$ the dechanneling length $L_{d}(\gamma, R)$ satisfies the relation 25

$$
L_{d}(\gamma, R)=\left(1-x^{2}\right)^{2} L_{d}(\gamma, \infty) \equiv\left(1-\frac{R_{c}}{R}\right)^{2} L_{d}(\gamma, \infty)
$$

where $L_{d}(\gamma, \infty)$ is the dechanneling length of a positron of the same energy in a straight channel $(R=\infty)$ and

$$
R_{c}=\frac{\varepsilon}{e U_{\max }^{\prime}}
$$

is the critical (minimal) radius consistent with the channeling condition in a bent crystal, "the centrifugal force $<$ the interplanar force" (2).

In an acoustically bent crystal the curvature $1 / R(z)$ is not constant (see (35)). Therefore it is natural to consider the mean curvature $1 / \bar{R}$ which is obtained by averaging $1 /|R(z)|$ over the undulator period

$$
\frac{1}{\bar{R}}=\frac{1}{\lambda} \int_{0}^{\lambda} k^{2} a|\sin k z| \mathrm{d} z=\frac{2}{\pi} \frac{1}{R_{\min }}
$$

with $R_{\min }$ from (38).

To proceed further we make use of the fact, that for a given crystal and crystallographic plane the reduced dechanneling length, $\alpha \equiv L_{d}(\gamma, \infty) / \gamma$, depends on $\gamma$ weakly. Its explicit expression, calculated by using the Lindhard approximation for the potential of a planar channel, reads [25]

$$
\alpha(\gamma)=\frac{256}{9 \pi^{2}} \frac{a_{\mathrm{TF}}}{r_{\mathrm{cl}}} \frac{d}{\ln (2 \varepsilon / I)-1}
$$


Here $a_{\mathrm{TF}}=0.8853 Z_{c}^{-1 / 3} a_{0}$ and $I=16 Z_{c}^{0.9} \mathrm{eV}$ are the Thomas-Fermi atomic radius and ionization potential, respectively. $Z_{c}$ is the atomic number of the crystal atoms, and $a_{0}$ is the Bohr radius. The dependences $\alpha(\gamma)$ for various planar channels are illustrated in Figure 11. For the $L i H$ crystal we used the average atomic number $Z_{c}=2$, and the interplanar spacings $d$ for its (100), (110) and (111) channels were deduced from 43.].

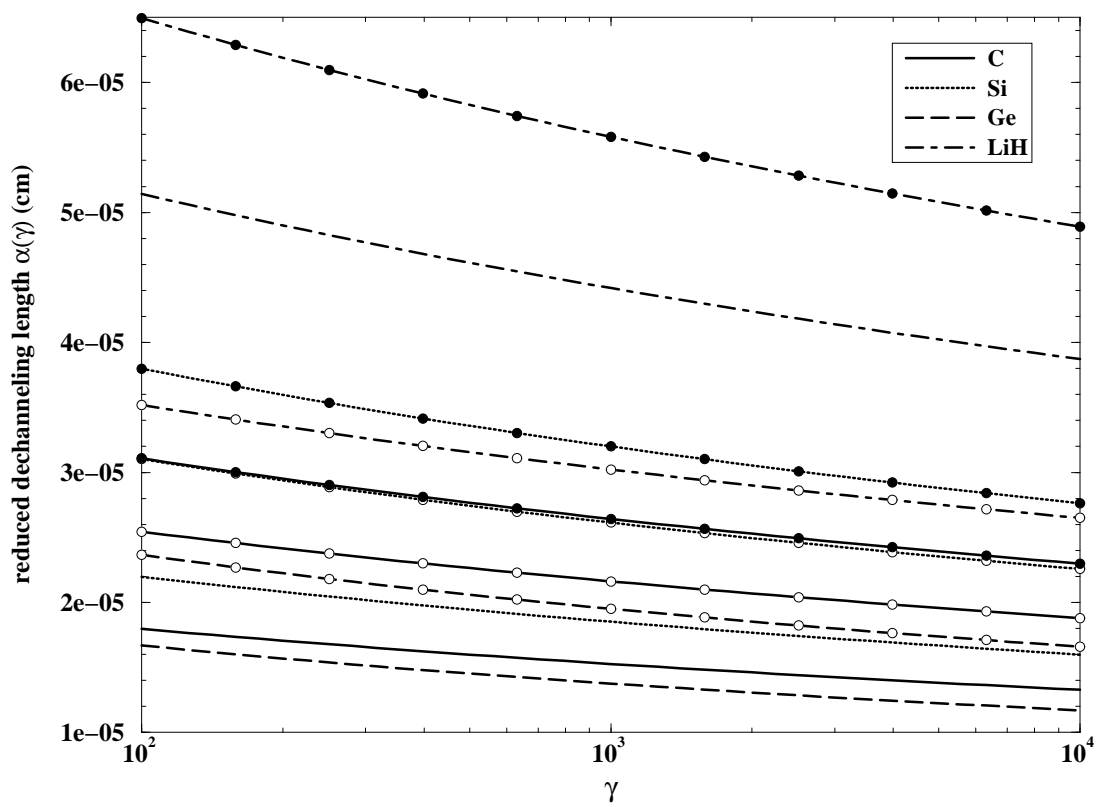

Figure 11. Dependence of a straight channel reduced dechanneling length, defined as $\alpha \equiv L_{d}(\gamma, \infty) / \gamma($ see also (62)), on the relativistic factor $\gamma$ of a positron channelled in (100), (110) and (111) planar channels of $C, S i, W$ and $L i H$ crystals as indicated. The full-circle curves correspond to (111) channels, the open-circle curves to (110), the curves without circles stand for (100) channels. The analogue curves for the Ge crystal (not presented in the figure) almost coincide with those for the $C$.

By introducing (59) and (52) in (56), and expressing $k$ through $x, R_{c}$ and $a$ as $k=x \sqrt{\pi / 2 R_{c} a}$, one gets

$$
g\left(L_{d}\right)=(2 \pi)^{2} \sqrt{\frac{\pi}{2}} \Theta(p) r_{\mathrm{cl}} n \frac{\alpha^{2}}{\gamma} \sqrt{\frac{e U_{\max }^{\prime}}{\varepsilon a}} x \cdot\left(1-x^{2}\right)^{4}
$$

This expression conveniently exposes the dependences of the gain factor on the projectile energy, on the parameters of a crystal, $U_{\max }^{\prime}$ and $\alpha$, and on the AW amplitude $a$. The factor $x \cdot\left(1-x^{2}\right)^{4}$ contains the dependence of $g\left(L_{d}\right)$ on the average curvature radius of the channel since $x^{2}$ is defined as $x^{2}=R_{c} / \bar{R}$.

By analysing (63) one obtains the ranges of parameters inside which the process of the emission stimulation occurs most efficiently. The factor $x \cdot\left(1-x^{2}\right)^{4}$ reaches its 
maximum value of 0.208 at

$$
x_{0}^{2}=\frac{\varepsilon}{e U_{\max }^{\prime} \bar{R}}=\frac{1}{9},
$$

defining, thus, for given $\gamma$ and crystal, the optimal value of a minimum curvature radius of the channel bent by the AW (recall (38)):

$$
R_{\min }^{o p t}=\frac{18}{\pi} \frac{e U_{\max }^{\prime}}{\varepsilon}
$$

Our analysis of an undulator based on an acoustically bent channel assumes the validity of the condition (11). It follows then, that the range of the $a$-values, being subject to $(64 a)$ ), is restricted from below to some $a_{\min } \gg d$.

Another condition to be fulfilled follows from (41) and implies that the total number of the undulator periods on the lengthscale $\left[L_{d}(\gamma, \bar{R})\right]_{x=x_{0}}=\left(1-x_{0}^{2}\right)^{2} L_{d}(\gamma, \infty)=$ $0.79 \cdot L_{d}(\gamma, \infty)$ is large. From (59), (60) and (64a) it follows then

$$
N_{\mathrm{u}}=\frac{0.79 x_{0}}{2 \sqrt{2 \pi}} \frac{\alpha \gamma}{\sqrt{R_{c} a}} \geq N_{\min } \gg 1
$$

This inequality defines the upper limit of the AW amplitude:

$$
a \leq a_{\max }=\frac{\pi}{2}\left(\frac{0.79 x_{0}}{2 \pi}\right)^{2} \frac{\alpha^{2} \gamma^{2}}{R_{c} N_{\min }^{2}}
$$

The inequalities $a_{\min } \gg d$ and (66) must be satisfied simultaneously, leading to a natural condition $a_{\min } \leq a_{\max }$. The latter results in the relation

$$
\alpha \gamma^{2} \geq \frac{2}{\pi}\left(\frac{0.79 x_{0}}{2 \pi}\right)^{2} \frac{m c^{2}}{e U_{\max }^{\prime}} N_{\min }^{2} a_{\min },
$$

giving the lowest value $\gamma_{\min }$ (which is obtained from (67)) with the equality sign). It allows to obtain the non-zero mesh defined by (64a).

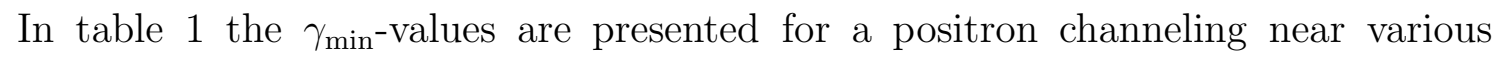
crystallographic planes in $C, S i, G e, W$ and $L i H$ crystals. The data correspond to $a_{\min }=10 d, N_{\min }=10$. The $d$ values for $C, S i, G e, W$ were taken from [25, 31], the $\mathrm{LiH}$ data were adopted from 43. The values of $e U_{\max }^{\prime}$ were calculated by using the Molière approximation [15] for an interplanar potential at the temperature $T=150 \mathrm{~K}$.

Thus, for given $\gamma \geq \gamma_{\min }$ the interval $\left[a_{\min }, a_{\max }\right]$ together with the values of $\nu$ following from (64b) and (39), represent the ranges of the AW amplitudes and frequencies whithin which the emission stimulation occurs with maximal efficiency and, simultaneously, the physical conditions (11), (39) and (41) are fulfilled. For these $a$ and $\nu$ values the expression for $g$ is given by

$$
\left[g\left(L_{d}\right)\right]_{x=x_{0}}=10.3 r_{\mathrm{cl}} n \frac{\Theta(p)}{\sqrt{a}} \frac{\alpha^{2}}{\gamma \sqrt{R_{c}}}
$$

Here the coefficient $10.3=(2 \pi)^{2} \sqrt{\pi / 2} \cdot x_{0}\left(1-x_{0}^{2}\right)^{2}$. 
Table 1. The values of $\gamma_{\min }$ (see (67)) calculated for a positron channelling in (100), (110) and (111) planar channels in $\mathrm{C}, \mathrm{Si}, \mathrm{Ge}, \mathrm{W}$ and $\mathrm{LiH}$ crystals. The minimum AW amplitude, $a_{\min }$, is set to $10 d$ ( $d$ is the interplanar distance). $e U_{\max }$ stands for a maximum gradient of the interplanar potential. $L_{d}\left(\gamma_{\min }, \bar{R}\right)$ and $\bar{R}$ are, respectively, the dechanneling length and the average bending radius, both corresponding to $\gamma_{\min }$.

\begin{tabular}{rrrrrr}
\hline Channel & $\begin{array}{r}d \\
\AA\end{array}$ & $\begin{array}{r}e U_{\max }^{\prime} \\
\mathrm{GeV} / \mathrm{cm}\end{array}$ & $\gamma_{\text {min }}$ & $\begin{array}{r}\bar{R} \\
\mathrm{~mm}\end{array}$ & $\begin{array}{r}L_{d}\left(\gamma_{\min }, \bar{R}\right) \\
\mathrm{mm}\end{array}$ \\
\hline $\mathrm{C}$ & & & & & \\
$(100)$ & 0.89 & 4.8 & 1570 & 1.68 & 0.184 \\
$(110)$ & 1.26 & 7.4 & 630 & 0.43 & 0.111 \\
$(111)$ & 1.54 & 9.5 & 380 & 0.20 & 0.084 \\
\hline $\mathrm{Si}$ & & & & & \\
$(100)$ & 1.36 & 4.8 & 1650 & 1.76 & 0.223 \\
$(110)$ & 1.92 & 7.1 & 700 & 0.51 & 0.148 \\
$(111)$ & 2.35 & 8.8 & 430 & 0.25 & 0.115 \\
\hline $\mathrm{Ge}$ & & & & & \\
$(100)$ & 1.41 & 9.6 & 1200 & 0.63 & 0.142 \\
$(110)$ & 2.00 & 14.0 & 510 & 0.18 & 0.091 \\
$(111)$ & 2.45 & 17.3 & 310 & 0.09 & 0.071 \\
\hline $\mathrm{W}$ & & & & & \\
$(100)$ & 1.58 & 39.8 & 330 & 0.04 & 0.039 \\
$(110)$ & 2.45 & 56.9 & 140 & 0.01 & 0.025 \\
\hline $\mathrm{LiH}$ & & & & & \\
$(100)$ & 1.90 & 1.8 & 1000 & 3.02 & 0.360 \\
$(110)$ & 1.30 & 1.0 & 300 & 15.50 & 0.675 \\
$(111)$ & 2.40 & 2.4 & 560 & 1.20 & 0.026 \\
\hline
\end{tabular}

Let us now define the amplitude $a_{0} \in\left[a_{\min }, a_{\max }\right]$, for which the gain factor (68) reaches its maximum. This quantity strongly depends on the factor $\Theta(p) / \sqrt{a}$, with $\Theta(p)$ from (57):

$$
\frac{\Theta(p)}{\sqrt{a}}= \begin{cases}1 / \sqrt{a} & \text { for } p^{2} \geq 1 \\ \frac{\pi x_{0}^{2} \gamma}{2 R_{c}} \cdot \sqrt{a} & \text { for } p^{2}<1\end{cases}
$$

The latter relation is obtained by replacing $k$ in $p=\gamma k a$ with $k=x_{0} \sqrt{\pi / 2 R_{c} a}$ (see (640) together with (39)).

It follows from (69) that the maximum point is at $a_{0}=a_{\min }$ if $p^{2} \geq 1$ for all $a$ from the interval $\left[a_{\min }, a_{\max }\right]$, and $a_{0}=a_{\max }$ if in the whole interval of $a$ the inequality $p^{2}<1$ is valid. A third option appears if the curves representing the dependencies $\gamma k a=1$ 
and $k^{2} a=\pi / 2 x_{0}^{2} R_{c}^{-1}$ cross in the point

$$
\tilde{a}=\frac{2 R_{c}}{\pi x_{0}^{2} \gamma^{2}}
$$

which lies within the interval $\left[a_{\min }, a_{\max }\right]$. In this case $a_{0}=\gamma^{-2}\left(2 / \pi x_{0}^{2}\right) R_{c}$.

Therefore, the quantity

$$
a_{0}= \begin{cases}a_{\min } & \text { if } \tilde{a}<a_{\min } \\ \tilde{a} & \text { if } a_{\min } \leq \tilde{a} \leq a_{\max } \\ a_{\max } & \text { if } a_{\max }<\tilde{a}\end{cases}
$$

defines the AW amplitude at which the gain achieves its maximum value which is:

$$
\left[g\left(L_{d}\right)\right]_{\max }=4.3 r_{\mathrm{cl}} n \frac{\alpha^{2}}{R_{c}} \times \begin{cases}\sqrt{\frac{\tilde{a}}{a_{\min }}} & \text { if } \tilde{a}<a_{\min } \\ 1 & \text { if } a_{\min } \leq \tilde{a} \leq a_{\max } \\ \sqrt{\frac{a_{\max }}{\tilde{a}}} & \text { if } a_{\max }<\tilde{a}\end{cases}
$$

The first harmonic frequency corresponding to the maximum gain (72), is obtained from relation(55), by expressing $\omega_{0}$ and $p^{2}$ through $a_{0}$ and $\tilde{a}$. The result for the photon energy, measured in $M e V$ reads

$$
\hbar \omega(\mathrm{MeV})=3.3 \cdot 10^{-11} \frac{\gamma^{2}}{\sqrt{R_{c} a_{0}}} \frac{1}{2+\left(a_{0} / \tilde{a}\right)^{2}}
$$

Figure 12 illustrates the dependence of the optimal AW amplitude $a_{0}$ (the diamondsembedded curves) and of $\hbar \omega$ (the circles-embedded curves) on $\gamma \geq \gamma_{\text {min }}$ in the case of a positron channeling near the (100), (110) and (111) planes in a diamond taken at temperature $T=150 \mathrm{~K}$. The curves corresponds to the particular values of $a_{\min }=10 \mathrm{~d}$ (see table 1) and $N_{\min }=10$. For other crystals the dependencies are basically the same, differing (not radically) only in ranges of the parameters.

The curves in figure 12 clearly demonstrate, that it is meaningful to consider the stimulated emission of high energy photons, $\hbar \omega \sim 0.01 \ldots 10 \mathrm{MeV}$, during the channeling of $\varepsilon \sim 0.1 \cdots 10 \mathrm{GeV}$ positrons in a crystal bent by a high-amplitude $(a \gg d)$ transverse acoustic wave propagating along the channel axis.

The above consideration focused on establishing the role of the dechanneling effect in the process of stimulated emission. The main restriction due to the dechanneling concerns the length of an undulator, which must be less than the dechanneling length, $L \leq L_{d}$. This, in turn, results in narrowing the ranges of $\gamma$ (eq. (67)), a (eq. (66)) and $\nu$ for which stimulated emission can effectively occur.

As it will be demonstrated below, small values of the undulator length, and, additionally, the attenuation of the photon flux in a crystal, both require high positron 


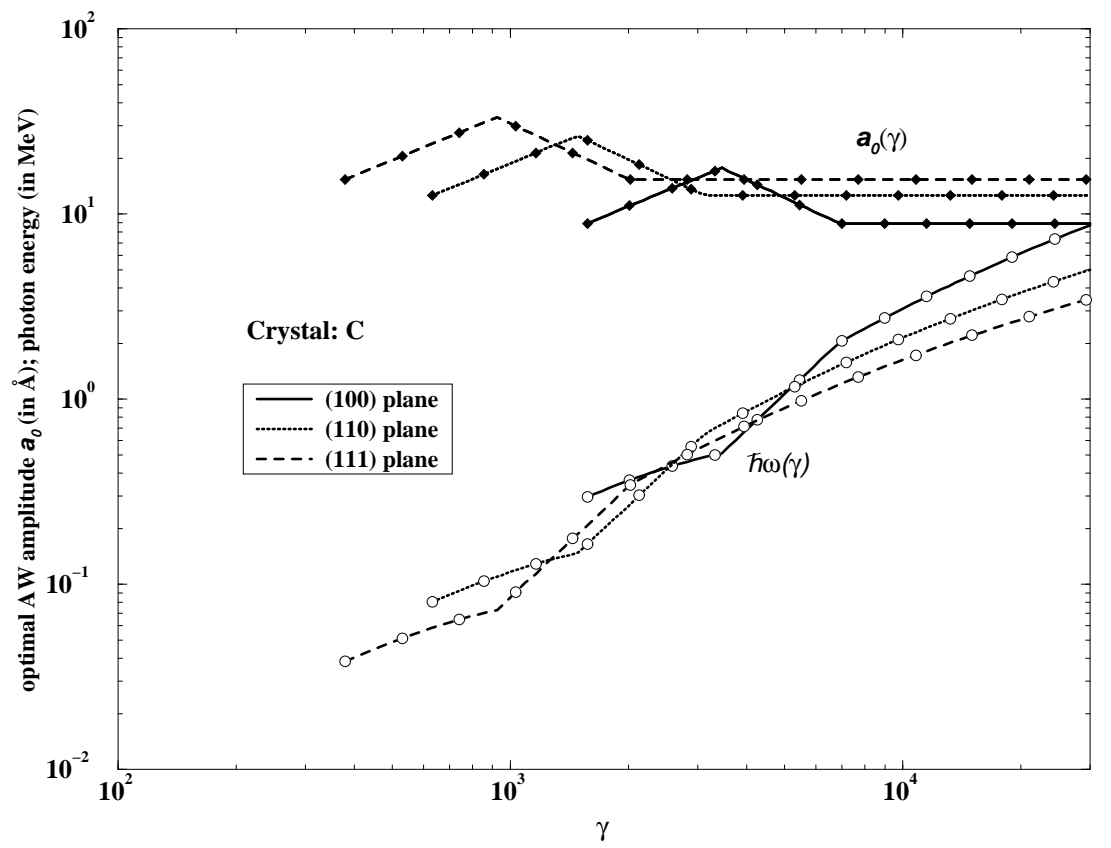

Figure 12. The magnitudes of the maximum gain acoustic wave amplitude (in $\AA$ ), $a_{0}$ (see (71)), and the first harmonic photon energy (in $\mathrm{MeV}$ ), $\hbar \omega$ (see (73)), versus the relativistic factor of a positron channeling in diamond (100), (110) and (111) planar channels (as indicated).

beam densities necessary to achieve a noticeable level of emission amplification. From the theory of FEL it is known that a model which considers a positron bunch as a group of independent particles (which has been utilized above) is adequate provided the following inequality is valid [61, 37]:

$$
\omega_{p} \tau<\gamma^{3 / 2}
$$

where $\omega_{p}=\left(4 \pi n \mathrm{e}^{2} / m\right)^{1 / 2}$ is the beam plasma frequency, and $\tau=L / c$ is the time of flight of the bunch through the crystal. The physical meaning of (74) is that $\tau$ is much smaller then the timescale needed to develop collective instabilities within the bunch due to its interaction with the undulator field and that of the radiation [37]. Therefore, all effects due to the space-charge dynamics during the channeling can be neglected if the volume density of the beam particles satisfies the condition

$$
n<\frac{1}{4 \pi r_{\mathrm{cl}}} \frac{\gamma^{3}}{L_{d}^{2}(\gamma, \bar{R})}
$$

Recalling that $L_{d}(\gamma, \bar{R})=\left(1-x_{0}^{2}\right)^{2} \gamma \alpha$, and inserting the values for the reduced dechanneling length $\alpha$ from figures 5.2, one obtains from (75) an estimation for $n$ in the range of $\gamma \approx 10^{3} \ldots 10^{4}: n<10^{22} \ldots 10^{23} \mathrm{~cm}^{-3}$.

Next we consider photon attenuation. Its influence can be accounted for in a rather 
simple way by suggesting that the increase per $1 \mathrm{~cm}$ in the total number of the emitted photons is given by

$$
\mathrm{d} N=(g-\mu) N \mathrm{~d} z
$$

rather than by (48). Here the quantity $\mu \equiv \mu(\omega)$ stands for the mass attenuation coefficient. These mass attenuation coefficients are tabulated for all elements and for a wide range of photon frequencies. For $\hbar \omega>1 \mathrm{KeV}$ and up to $100 \mathrm{MeV}$ the $\mu$-values can be found in ref. [54]. Figure 13 shows the $\mu(\omega)$ dependences for various crystals. The step-like behaviour of the curves for $S i, G e$ and $W$ is due to the discontinuity of the atomic photoeffect cross section in the vicinity of inner-shell thresholds. For photons of energy less than $1 \mathrm{KeV}$ the main contribution to the photon attenuation arises from the atomic photoeffect process, and the $\mu(\omega)$ dependence is easily deduced from ref. [59].

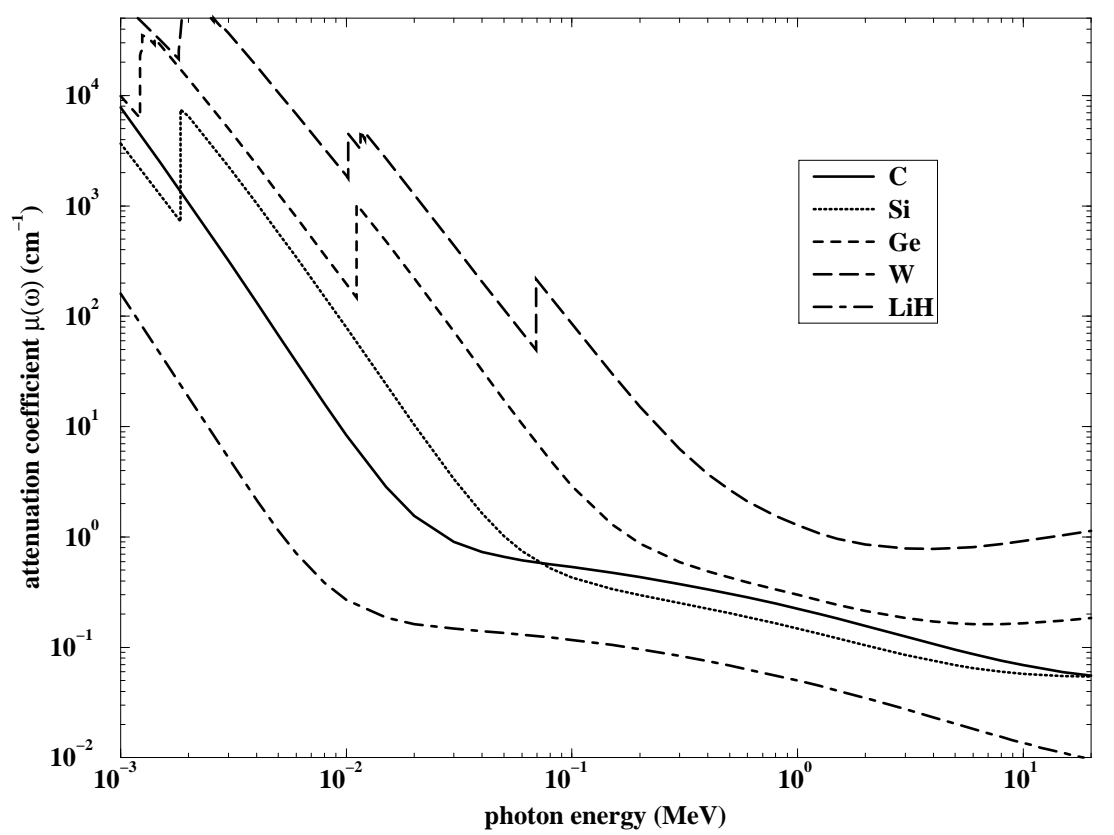

Figure 13. The mass attenuation coefficient, $\mu$, in dependence on photon energy for various crystals, as indicated. The data are taken from [54.

To provide an increase in the radiation intensity the gain must satisfy the condition

$$
g>\mu(\omega)
$$

which leads to further narrowing of the interval of photon energies. The large magnitude of $\mu(\omega)$ for $\hbar \omega<10 \mathrm{KeV}$ (see figure 13) makes it almost impossible to obtain stimulated emission in this $\omega$-region even for crystals with small average atomic number.

To draw a conclusion on what type of a crystal and a plane is more suitable from the viewpoint of its efficiency to amplify the spontaneous emission formed in an acoustically 
based undulator let us make qualitative estimates of the density of a positron beam needed to achieve the magnitude of 1 for the total gain.

The total gain, $G$, which represents by itself the total increase in the number of photons due to the stimulated emission on the lengthscale $L \equiv\left[L_{d}(\gamma, \bar{R})\right]_{x=x_{0}}$, equals

$$
G=(g(L)-\mu(\omega)) L
$$

Such a definition of the total gain is valid in the low-gain limit, $G \ll 1$ (see e/g. [31, 37]). Nevertheless, for estimation purposes, one may use (78) in the region $G \leq 1$.

Putting $G=1$ and representing the quantity $g(L)$ in the form $g(L)=\tilde{g}(L) \cdot n$ (the explicit expression for $\tilde{g}(L)$ is clear from (68)), we reach the following estimate for the volume density of beam particles:

$$
n=\frac{1+\mu(\omega) L}{\tilde{g}(L) L}
$$

The dependences $n$ versus $\omega$ for various energies of a positron beam and for various types of planar channels (as indicated) are presented in figures 14. For each channel and for $\varepsilon \geq \varepsilon_{\min }=m c^{2} \gamma_{\min }$ (the definition of $\gamma_{\min }$ is given by (67) and its particular values are presented in table 1) the quantity $\tilde{g}(L)$ can be calculated from (68)), and the corresponding $\omega$ range was calculated by using the relation

$$
\hbar \omega(\mathrm{MeV})=3.3 \cdot 10^{-11} \frac{\gamma^{2}}{\sqrt{R_{c} a}} \frac{1}{2+(a / \tilde{a})^{2}}
$$

where $\tilde{a}$ is defined in (70). In (80) it is assumed that the AW amplitude $a$ varies within the interval $a=\left[a_{\min }, a_{\max }\right]$, and both $a$ and the AW frequency $\nu$ are subject to (64b). The minimum value of the AW amplitude was chosen as $a_{\min }=10 d$, the quantity $a_{\max }$ was calculated from (66) with the munimum number of the undulator periods $N_{\text {min }}=10$. The magnitudes of the mass attenuation coefficients, $\mu(\omega)$, were obtained by interpolating the data from [54].

For each curve in figures 14 the minimum value of the beam density is achieved at $a=a_{0}$ (see (71)) with the corresponding magnitude of the photon energy given by (73). The only exception is the $n(\omega)$ dependence for a $250 \mathrm{MeV}$ positron beam channeling along the (100) plane in $W$ (see figure 14(a)). In this case, due to the irregularity in the behaviour of $\mu(\omega)$ in the vicinity of the $1 s$ atomic threshold $\left(I_{1 s}=69.5 \mathrm{keV}\right.$ for $\left.W\right)$,see figure 13, - the minimum of the beam density is achieved at $\hbar \omega \approx 70 \mathrm{keV}$.

Figure 15 illustrates the extent to which the photon attenuation influences the minimum value of the beam density. For each crystal (as indicated) the dependence $n(\omega)$ from $(79)$ is represented by the curves without full circles. The curves embedded with full circles correspond to the function $\tilde{n}(\omega)=[n(\omega)]_{\mu(\omega) \equiv 0}$, giving thus the minimum values of the volume density which is needed to achieve the total gain $G=1$ in the case when the photon attenuation is totally disregarded. It is seen that for $\hbar \omega \geq 1 \mathrm{MeV}$ 


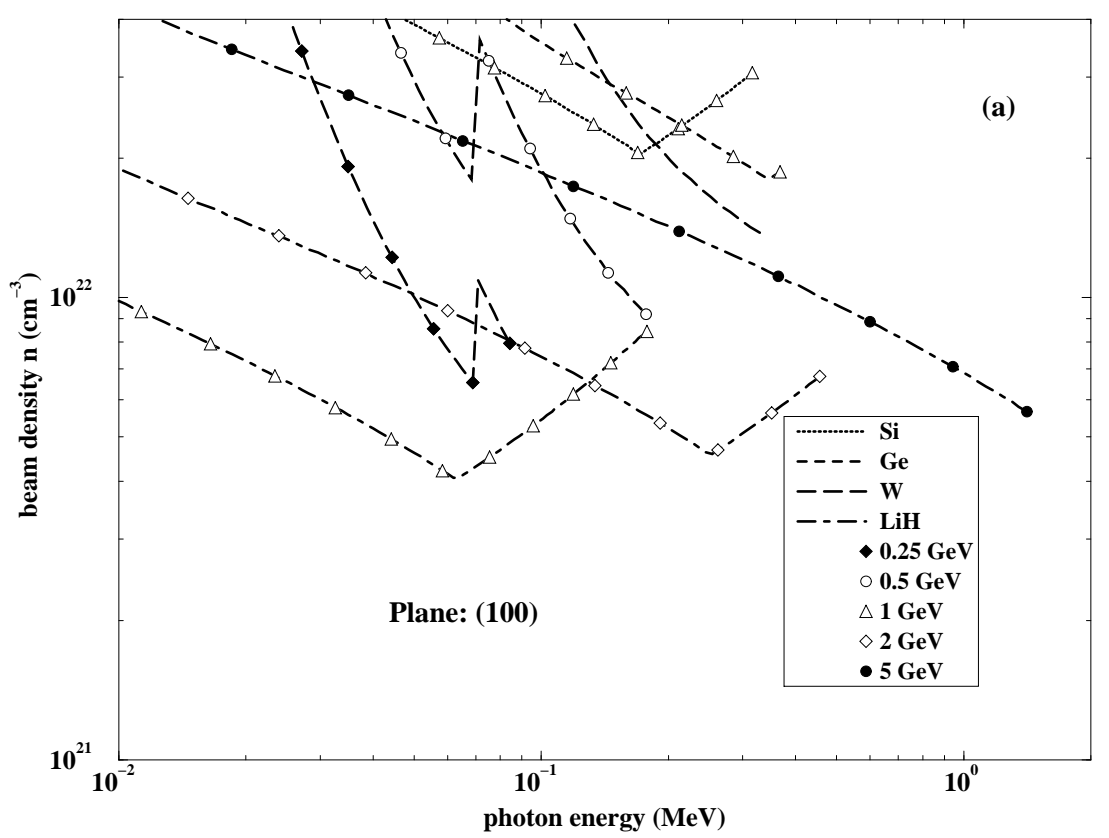

Figure 14. The volume density needed to achieve the total gain $G=1$ for a positron beam (for various energies $\varepsilon$ as indicated) channeling along (a) (100) plane, (b) (110) plane, (c) (111) plane, in various crystals as indicated. The curves correspond to (79).

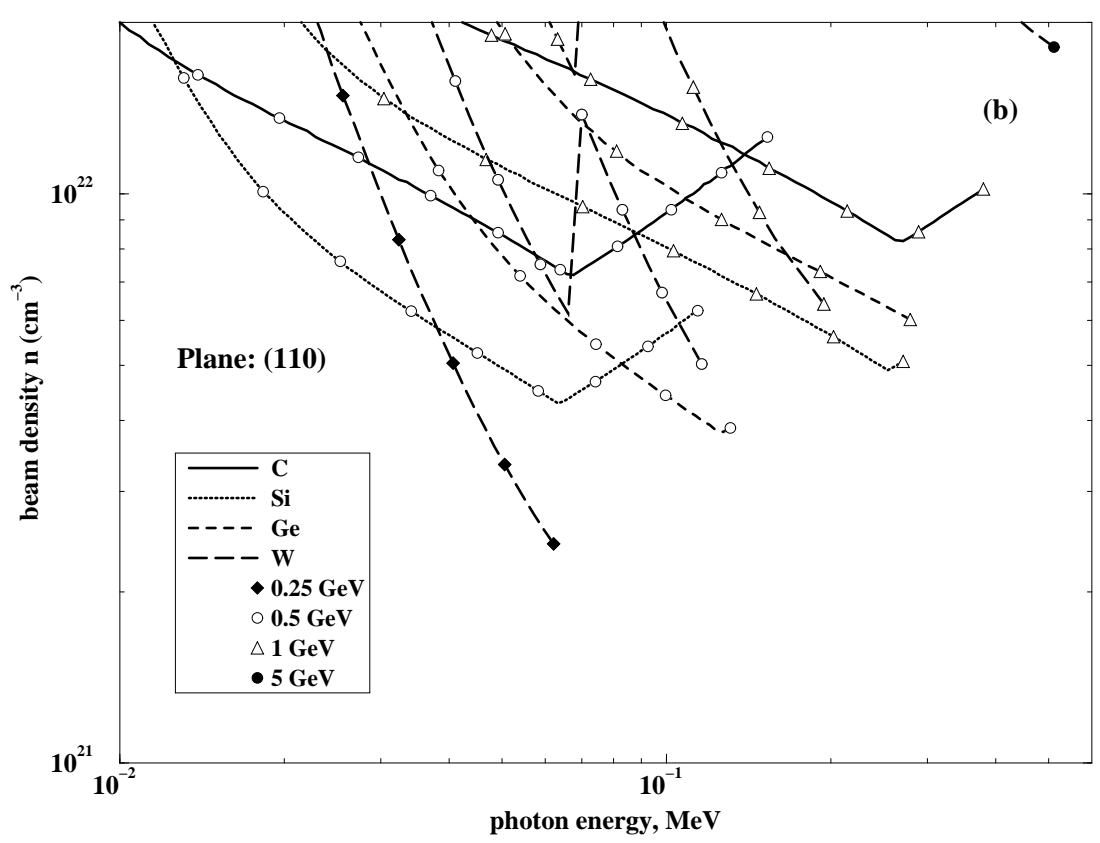

Figure 14. (continued) 


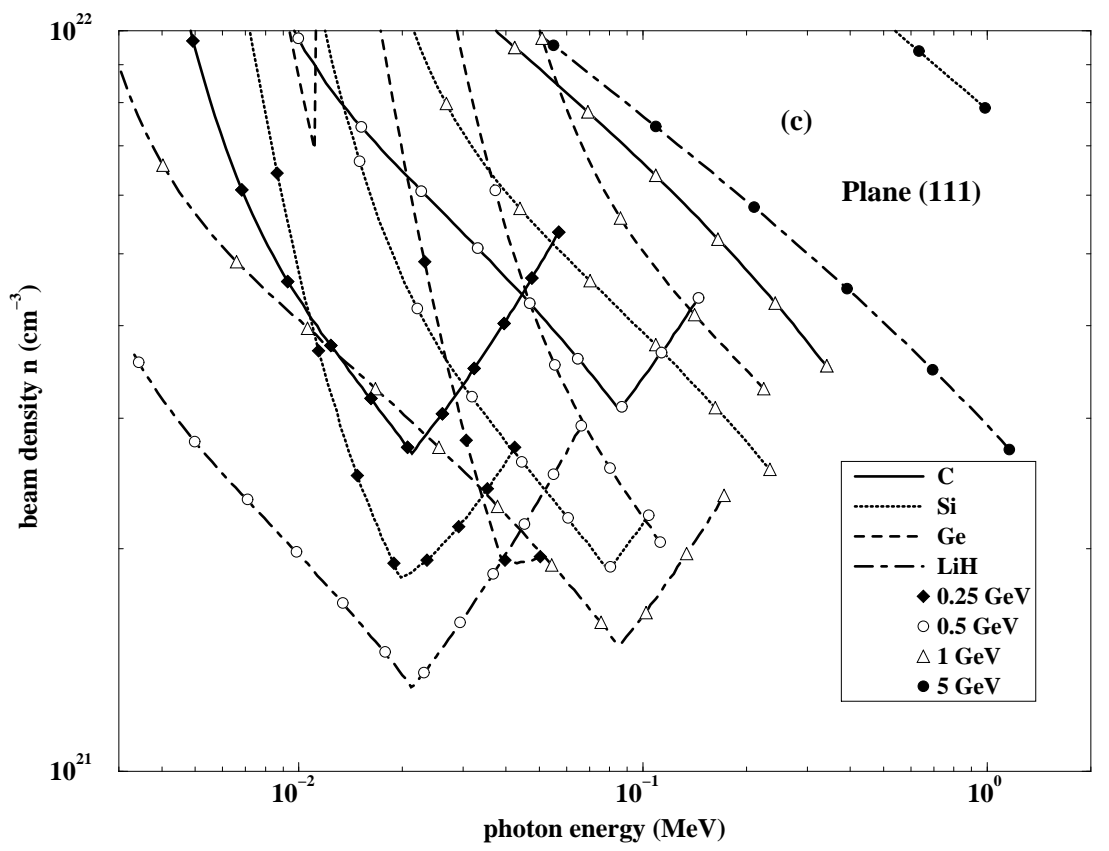

Figure 14. (continued)

the role of the attenuation is negligibly small for all crystals, whereas for less energetic photons it leads to a prominent increase in $n$.

Figures 14 and 15 show that to achieve a noticeable level of the amplification of the spontaneous radiation formed in the acoustically based undulator large magnitudes of a positron beam volume density, $n \sim 10^{21}-10^{22}$, are needed. This is mainly due to the dechanneling effect which imposes strong restrictions on the undulator length.

These values of $n$, being high enough, are, nevertheless comparable with those planned to be achieved within the TESLA project 62].

Therefore, we believe that the scheme described above may be considered as a new feasible source for stimulated emission within the photon energies range from tens of $\mathrm{KeV}$ up to $\mathrm{MeV}$ by means of the positron beam channeling in an acoustically bent crystal.

\section{Concluding remarks}

Our investigation shows that a crystal, which is periodically bent by a transverse acoustic wave, can be used for the construction of an undulator for a beam of ultra-relativistic particles channeled in the lattice. The parameters of this undulator can be tuned by varying the AW amplitude and frequency, the energy of the projectile, and by using different types of crystals and its channels. The suggested undulator can be used for the generation of the radiation of high energy photons. 


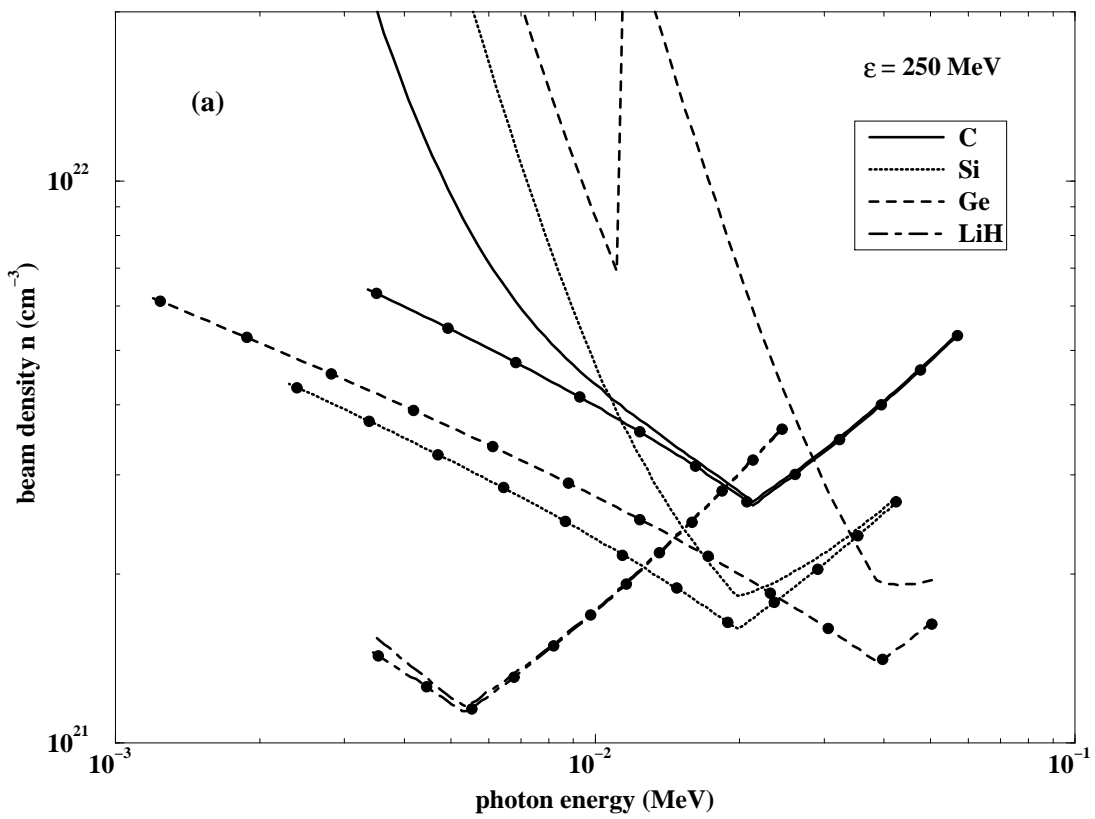

Figure 15. The volume density needed to achieve the total gain $G=1$ for a 500 $\mathrm{MeV}$ positron beam channeling near the (111) planar channels in various crystals as indicated. The curves without full circles corresponds to (79). The curves with full circles represent the $(79)$ dependences with $\mu(\omega) \equiv 0$ (i.e. no photon attenuation taken into account).

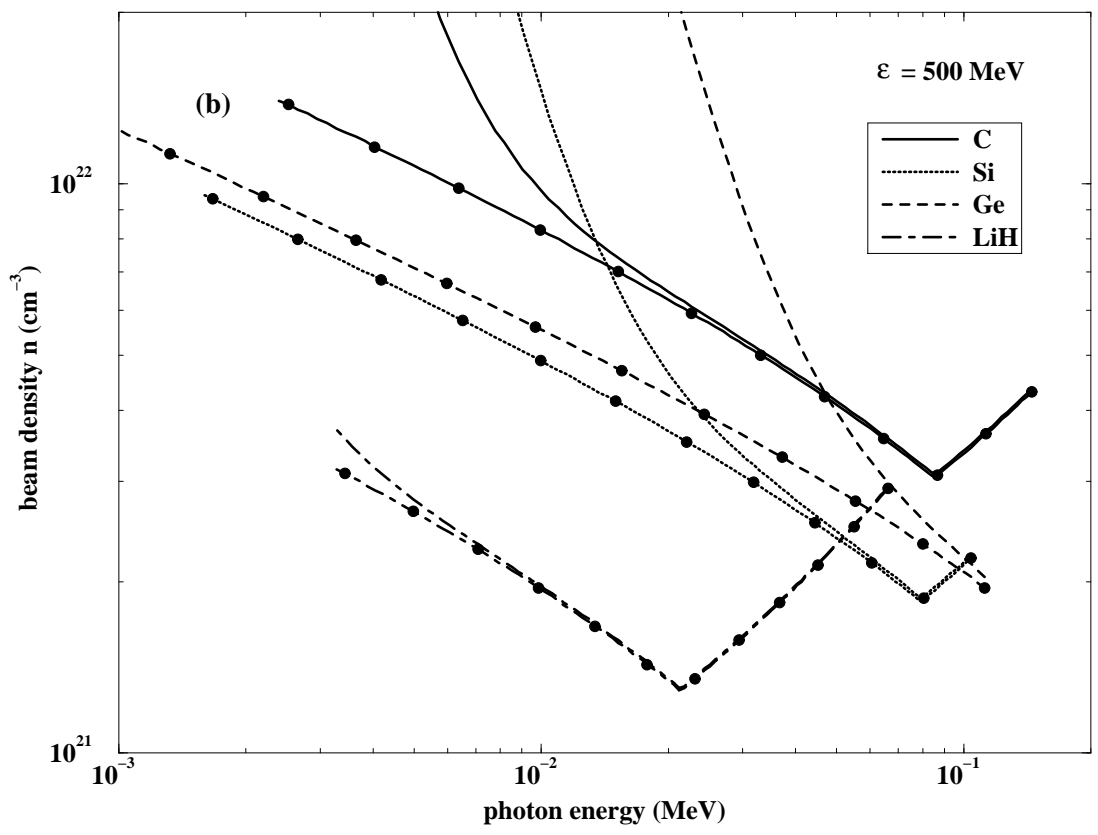

Figure 14. (continued) 
Also, it is shown that it is meaningful to discuss the possibility to create a powerful source of a free-electron laser type stimulated radiation in the energy range of tens of $\mathrm{keV}$ up to the $\mathrm{MeV}$ region.

We consider our present research as an initial milestone for more advanced theoretical treatment of the problem. The goal of this further investigation is to achieve more accurate quantitative description of the undulator radiation and the corresponding laser effect. In out opinion, on this way the following phenomena must be thoroughly considered.

1. The AIR mechanism for a wide beam. When the wide beam enters the crystal it is spread over many channels. Each channel is bent by the AW and, hence, one must (when calculating spontaneous and stimulated emission) take into account the photon fluxes formed by these sub-beams. In this case the coherence effect in the photon emission can be enhanced due to the interaction of the photons with the particles moving in many neighbouring channels. In the present paper we have investigated the undulator and the laser effects in a single channel.

2. Kinetics in the AIR problem. The more advanced description of the AIR problem must take into account the kinetic behaviour of the particles in the beam as well as kinetics of the photon flux. Such a description will treat properly the dechanneling mechanism, the attenuation phenomenon and the interaction of photons and particles beams.

3. Dechanneling in a periodically bent lattice. It is necessary to study dechanneling in a periodically bent channel in comparison with this effect in a straight channel and/or in a channel with constant (or/and slowly varied) curvature. So far nobody studied dechanneling in a periodically bent channel (the AW bending). The dechanneling length might increase as compared with the linear channel case. The dechanneling length plays the crucial role for the laser effect which we discuss in our paper (see Section 5). Indeed, increasing of the dechanneling length by factor of 10 results in decreasing of the channeling beam density, necessary for achievement of large gain $G$, by the factor of 1000 (see eqs. (56) and (79)).

Let us stress here that special attention must be paid the problem of dechanneling of a wide beam in a periodically bent crystal. Broadening of the channeled beam in such a medium, which was not considered before, is important in our problem.

4. The bunching effect. The effect of the stimulation of photon emission can be enhanced by increasing density $n$ of the channeling beam. From the theory of free electron lasers (e.g. 63) it is known that for high $n$ large gain factors can be achieved in the regime of collective instabilities in the beam (the bunching effect). In our future reaserch we plan to apply the results of the FEL theory to the description of the laser-type radiation based on the AIR phenomenon.

5. Acoustic waves of various configurations. In the present paper we investigated 
spontaneous and stimulated AIR in an acoustically bent crystal considering the most simple example of the acoustic wave, the monochromatic plane wave. However, other cases of AW (longitudinal waves, spherical waves, nonmonochromatic waves and various combinations thereof), interacting with the beam of the channeling particles, are worthy to study. By applying the more complex acoustic waves for the crystal bending one may construct an undulator with variable parameters for the generation of high energy photons in a wide range. The use of the crystal-based undulator with variable parameters may result in noticeable increase of the gain factor for the stimulated AIR analogously to how it occues in the free electron lasers based on the tampered magnetic wigglers [64].

6. Combination of the ordinary channeling radiation and the AIR. The present consideration was focused on the case of the high amplitude AW (see (1)). This allowed us to disregard the contribution of the ordinary channeling radiation to the total spectrum of the emitted photons. More general approach must include both radiative mechanisms, as well as their interference, simultaneously.

The rigorous treatment of the above outlined problems will be the subject of further publications.

\section{Acknowledgments}

We express our gratitude to Professor E. Uggerhøj for helpful discussion. The authors acknowledge support from the DFG, GSI, BMBF and, especially, from the Alexander von Humboldt Foundation.

\section{References}

[1] Korol A V, Solov'yov A V and Greiner W, Acoustically induced radiation of a charged particle channeling in a crystal, UFTP467/Preprint/1997, Institute for Theoretical Physics, Frankfurt am Main University.

[2] Korol A V, Solov'yov A V and Greiner W 1998 J.Phys.G.: Nucl. Part. Phys. 24 L45

[3] Baryshevsky V G, Dubovskaya I Ya and Grubich A O 1991 J. Phys. C: Cond. Matt. 2421

[4] Baryshevsky V G 1982 Channeling, Radiation and Reactions in Crystals at High Energies (Minsk: Buelorussian State University Press) p 256

[5] Ikezi H, Lin Liu Y R and Ohkawa T 1984 Phys. Rev. B 301567

[6] Bogacz S A and Ketterson J B 1986 J. Appl. Phys. 60177

[7] Mkrtchyan A R, Gasparyan R A and Gabrielyan R G Zh. Eksp. Teor. Fiz. 198793432 (Engl. transl. Sov. Phys. - JETP 198766 248)

[8] Baryshevsky V G and Dubovskaya I Ya 1991 J. Phys. C: Cond. Matt. 32421

[9] Dedkov G V 1994 Phys. Stat. Sol. (b) 184535

[10] Robinson M T 1962 Appl.Phys.Lett. 149

[11] Robinson M T and Oen O S 1963 Appl.Phys.Lett. 2 30; Phys.Rev. 1322385 
[12] Stark J 1912 Phys. Z. 13973

[13] Lehmann C and Leibfreid G 1963 J. Appl. Phys. 342821

[14] Lindhard J 1965 Kong. Danske Vid. Selsk. mat.-fys. Medd. 3414

[15] Gemmell D S 1974 Rev. Mod. Phys. 46129

[16] 1987 Relativistic Channeling ed A Carrigan and J Ellison (NY: Plenum)

[17] Sørensen A H and Uggerhøj E 1989 Nucl. Sci. Appl. 3147

[18] 1996 Channeling and other crystal effects at relativistic energy topical issue of Nucl. Instum. and Meth. in Phys. Res. Section B 119 ed H H Andersen and L E Rehn

[19] Tsyganov E N 1976 Fermilab preprint TM-682, 684 (Batavia)

[20] Elishev A F et al 1979 Phys. Lett. B 88387

[21] Bak J F , Melchart G, Uggerhøj E, Forster I S, Jensen P R, Mads Boll H, Møller S P, Nielsen N, Petersen G, Schiott H, Gross J J and Siffert P 1980 Phys. Lett. B 93505

[22] Møller S P , Uggerhøj E, Atherton H W, Clément M, Doble N, Elsener K, Gatignon L, Grafström P, Hage-Ali M and Siffert P 1991 Phys. Lett. B 25691

[23] Biino C, Clément M, Doble N, Elsener K, Freund A, Gatignon L, Grafström P, Kirsebom K, Mikkelsen U, Møller S P, Uggerhøj E and Worm T 1997 Phys. Lett. B 40391

[24] Arduini G,Biino C, Clément M, Cornelis K, Doble N, Elsener K, Ferioli G, Fideraco G, Gatignon L, Grafström P, Gyr M, Klem J, Mikkelsen U, Weisse E, Møller S P, Uggerhøj E, Tatarin A, Freund A, Keppler P and Major J 1997 Phys. Rev. Lett. 794182

[25] Biruykov V M, Chesnokov Y A and Kotov V I 1996 Crystal Channeling and its Application at High-Energy Accelerators (Berlin: Springer)

[26] Schäfer A and Greiner W 1991 J. Phys. G: Nucl. Part. Phys. 17 L217

[27] Solov'yov A V, Schäfer A and Greiner W 1996 Phys. Rev. E 531129

[28] Kumakhov M A 1976 Phys. Lett. A57 17

[29] Baryshevsky V G and Dubovskaya I Ya 1977 Phys. Stat. Sol. (b) 82403

[30] Bazylev V A and Zhevago N K 1990 Usp. Fiz. Nauk 16047 (Sov. Phys. - Uspekhi 331021 (1990)); 1987 Radiation of Fast Charged Particles in Matter and External Fields (Moscow: Nauka) p 270

[31] Baier V N, Katkov V M and Strakhovenko V M 1989 High Energy Electromagnetic Processes in Oriented Monocrystals (Novosibirsk: Nauka); 1997 High Energy Electromagnetic Processes in Oriented Single Crystals (Singapore: World Scientific)

[32] Kumakhov M A and Komarov F F 1989 Radiation From Charged Particles in Solids (New York: AIP)

[33] Arutyunov V A, Kudryashov N A, Samsonov V M and Strikhanov M N 1991 Zh. Tehn. Fiz. 61(2) 32; ibid. 61(3) 1; 1991 Nucl. Phys. B 363283

[34] Ginzburg V L 1947 Izv. AN SSSR 11165

[35] Motz H 1951 J. Appl. Phys. 222684

[36] Alferov D F, Bashmakov Yu A and Cherenkov P A 1989 Usp. Fiz. Nauk 157389 (Sov. Phys. Uspekhi 32200 (1989))

[37] Fedorov M V 1991 Electron in a Strong Light Field (Moscow: Nauka) (in Russian)

[38] Kumakhov M A and Trikalinos Kh G 1980 Zh. Eksp. Teor. Fiz. 781623 (Sov. Phys. - JETP 51 815 (1990))

[39] Colson W B 1997 Nucl. Inst. and Meth. A 3936

(see also http://www.physics.nps.navy.mil/fel.html)

[40] Ellison J A and Picraux S T 1981 Phys. Lett. A 83271 
[41] Baier V N and Katkov V M Zh. Eksp. Teor. Fiz. 196753 1478; 196855 1542; (Engl. transl. Sov. Phys. - JETP 196826 854; 196928 807)

[42] Berestetskii V B, Lifshitz E M and Pitaevskii L P 1982 Quantum Electrodynamics (Oxford: Pergamon)

[43] Berman B L, Kephart J O, Datz S, Klein R K, Pantell R H, Swent R L, Park H, Alguard M J and Hynes M V 1996 Nucl. Instrum. Meth. B 11971

[44] Landau L D and Lifshitz 1971 The Classical Theory of Fields (Oxford: Pergamon)

[45] Abramowitz M and Stegun I E 1964 Handbook of Mathematical Functions (New York: Dover)

[46] Mason W P 1972 Acoustic properties of Solids, American Institute of Physics Handbook, 3rd edn (New York: McGraw-Hill)

[47] Colson W B, Datolli G and Ciocci F 1985 Phys. Rev. A 31828

[48] Bonifacio R, Pellegrini C and Narducci L M 1984 Opt. Commun. 50373

[49] Pellegrini C 1985 Nucl. Instrum. Meth. A 239127

[50] Baier V N and Mil'shtein A I 1980 Dokl. Akad. Nauk SSSR 2501364 (Engl. transl. Sov. Phys. Dokl. 198025112

[51] Murphy J B and Pellegrini C 1985 Nucl. Instrum. Meth. A 237159

[52] Kwang-Je Kim and Ming Xie 1993 Nucl. Instrum. Meth. A 331359

[53] Saldin E L, Schneidmiller E A and Yurkov M V 1995 Phys. Rep. 260187

[54] Hubbel J H and Seltzer S M Tables of X-ray Mass Attenuation Coefficients and Mass EnergyAbsorption Coefficients $1 \mathrm{keV}$ to $20 \mathrm{MeV}$ for elements $Z=1$ to 92 and 48 Additional Substances of Dosimetric Interest NISTIR 5632 - Web Version 1.02 http://physics.nist.gov/PhysRefData/XrayMassCoef/cover.htm

[55] Kolomenski A A and Lebedev A N Kvantovaya Elektronika 51543

[56] Madey J M J 1971 J. Appl. Phys. 421906

[57] Coisson R J 1981 IEEE J. Quantum Electr. QE-17 1409

[58] Hopf F A, Meystre P, Scully M O and Louisell W H 1976 Opt. Comm. 18413

[59] Henke B L, Gullikson E M and Davis J C 1993 Atomic Data and Nuclear Data Tables 54 No 2; see also at http://xray.uu.se/hypertext/henke.htm

[60] Ternov I M, Khailov V R and Kholomai B V 1985 Zh. Eksp. Teor. Fiz. 88329 (Engl. transl. Sov. Phys. - JETP 198561 192)

[61] Louisell W H, Lam J F and Copeland D A 1978 Phys. Rev. A 18655

[62] 1997 Conceptual Design of a $500 \mathrm{GeV} e^{+} e^{-}$Linear Collider with Integrated X-ray Laser Facility vols I and II, ed R Brinkmann et al DESY 1997-048, ECFA 1997-182

[63] Bonifacio R, Casagrande F, Cerchioni G, de Salvo Souza L, Pierini P and Piovella N 1990 Rivista del Nuovo Cimento 13 No.9 1

[64] Kroll N M, Morton P L and Rosenbluth M N 1981 IEEE Journal Quant. Electr. QE-17 1436 